\title{
Long colimits of topological groups I: Continuous maps and homeomorphisms*
}

\author{
Rafael Dahmen and Gábor Lukács
}

November 28, 2019

\begin{abstract}
Given a directed family of topological groups, the finest topology on their union making each injection continuous need not be a group topology, because the multiplication may fail to be jointly continuous. This begs the question of when the union is a topological group with respect to this topology. If the family is countable, the answer is well known in most cases. We study this question in the context of so-called long families, which are as far as possible from countable ones. As a first step, we present answers to the question for families of group-valued continuous maps and homeomorphism groups, and provide additional examples.
\end{abstract}

\section{Introduction}

Given a directed family $\left\{G_{\alpha}\right\}_{\alpha \in \mathbb{I}}$ of topological groups with closed embeddings as bonding maps, their union $G=\bigcup_{\alpha \in \mathbb{I}} G_{\alpha}$ can be equipped with two topologies: the colimit space topology defined as the finest topology $\mathscr{T}$ making each map $G_{\alpha} \rightarrow G$ continuous, and the colimit group topology, defined as the finest group topology $\mathscr{A}$ making each map $G_{\alpha} \rightarrow G$ continuous. The former is always finer than the latter, which begs the question of when the two topologies coincide.

We say that $\left\{G_{\alpha}\right\}_{\alpha \in \mathbb{I}}$ satisfies the algebraic colimit property (briefly, $A C P$ ) if $\mathscr{T}=\mathscr{A}$, that is, if the colimit of $\left\{G_{\alpha}\right\}_{\alpha \in \mathbb{I}}$ in the category Top of topological spaces and continuous maps coincides with the colimit in the category Grp(Top) of topological groups and their continuous homomorphisms.

The family $\left\{G_{\alpha}\right\}_{\alpha \in \mathbb{I}}$ satisfies ACP if and only if $(G, \mathscr{T})$ is a topological group. In general, the inversion $(G, \mathscr{T}) \rightarrow(G, \mathscr{T})$ is continuous, and the multiplication $m:(G, \mathscr{T}) \times(G, \mathscr{T}) \rightarrow(G, \mathscr{T})$ is separately continuous, but need not be jointly continuous. Thus, $\left\{G_{\alpha}\right\}_{\alpha \in \mathbb{I}}$ satisfies ACP if and only if $m$ is continuous.

It is well known that ACP holds if: (1) the bonding maps are all open; or (2) $I$ is countable and each $G_{\alpha}$ is locally compact Hausdorff ([10, Theorem 4.1] and [8, Propositions 4.7 and 5.4]). Yamasaki showed that for countable families of metrizable groups, these two are the only cases where ACP holds.

*2010 Mathematics Subject Classification: Primary 22A05, 46M40; Secondary 22F50, 46E40, 54C35. 
Theorem 1.1. ([15, Theorem 4]) Let $\left\{G_{n}\right\}_{n \in \mathbb{N}}$ be a countable family of metrizable topological groups with closed embeddings as bonding maps. If

(a) there is $n$ such that $G_{n}$ is not locally compact, and

(b) for every $n$ there is $m>n$ such that $G_{n}$ is not open in $G_{m}$,

then the colimit space topology $\mathscr{T}$ is not a group topology on $G=\bigcup G_{n}$, that is, $\left\{G_{n}\right\}_{n \in \mathbb{N}}$ does not satisfy ACP.

While ACP is well understood for countable families of metrizable groups, Yamasaki's theorem does not hold in the uncountable case. For example, if $\mathbb{I}=\omega_{1}$ and the bonding maps are isometric embeddings, then ACP holds (Corollary 2.11). Similarly, if $\mathbb{I}=\omega_{1}$ and $G_{\alpha}=\prod_{\beta<\alpha} M_{\beta}$, where each $M_{\alpha}$ is metrizable, then ACP holds, and the resulting group is the $\Sigma$-product of the $M_{\beta}$ (Corollary 2.6).

Motivated by these examples, the aim of the present paper is to examine directed families that are as far as possible from countable ones. A directed set $(\mathbb{I}, \leq)$ is long if every countable subset of $\mathbb{I}$ has an upper bound in $\mathbb{I}$. It may be tempting to conjecture that all long families of topological groups satisfy ACP; this, however, is not the case (see Example 2.15). Nevertheless, long families provide a number of interesting examples where ACP holds.

We say that a Hausdorff space $X$ is a long space if the set $\mathscr{K}(X)$ of compact subsets of $X$ ordered by inclusion is long, that is, every $\sigma$-compact subset of $X$ has a compact closure. The product $\mathbb{L}_{\geq 0}:=\omega_{1} \times[0,1)$ equipped with order topology generated by the lexicographic order is called the Closed Long Ray. The Long Line $\mathbb{L}$ is obtained by gluing together two copies of the Closed Long Ray, one with the reverse order and one with the usual order, at the boundary points $(0,0)$. The connected spaces $\mathbb{L}_{\geq 0}$ and $\mathbb{L}$ share many of the properties of the zero-dimensional space $\omega_{1}$ : they are locally compact Hausdorff, first countable, and normal, but neither metrizable nor paracompact. Both $\mathbb{L}_{\geq 0}$ and $\mathbb{L}$ are long spaces, and any cardinal of uncountable cofinality with the order topology is a long space.

For a topological space $X$ and a topological group $M$, we put $C_{c p t}(X, M)$ for the set of continuous compactly supported $M$-valued functions on $X$, where the support of $f: X \rightarrow M$ is $\operatorname{cl}_{X}\left\{x \in X \mid f(x) \neq e_{M}\right\}$. We equip $C_{c p t}(X, M)$ with pointwise operations and the uniform topology. The next theorem shows that for a long space $X$, the uniform topology is the "natural" one on $C_{c p t}(X, M)$.

Theorem A. Let $X$ be a long space and $M$ be a metrizable group. For $K \in \mathscr{K}(X)$, let $C_{K}(X, M)$ denote the subgroup of $C_{c p t}(X, M)$ consisting of functions with support in $K$. Then the family $\left\{C_{K}(X, M)\right\}_{K \in \mathscr{K}(X)}$ satisfies $A C P$ and

$$
\underset{K \in \mathscr{K}(X)}{\operatorname{colim}} C_{K}(X, M)=C_{c p t}(X, M) .
$$

Recall that a space $X$ is pseudocompact if every continuous real-valued map on $X$ is bounded. One says that $X$ is $\omega$-bounded if every countable subset of $X$ is contained in a compact subset. Long spaces have previously been studied under the names strongly $\omega$-bounded and $\sigma C$-bounded ([13] and [12]). The relationship between a Hausdorff space $X$ being a long space and other compactness-like properties is as follows:

$$
\text { compact } \Longrightarrow \text { long } \Longrightarrow \omega \text {-bounded } \Longrightarrow \text { pseudocompact, }
$$


and for metrizable spaces they are all equivalent. Nyikos showed that an $\omega$-bounded space need not be long ([13]). The next theorem shows that the requirement of $X$ being long cannot be omitted from Theorem $\mathrm{A}$,

Theorem B. Let $X$ be a locally compact Hausdorff space and $V$ a non-trivial metrizable topological vector space. If $X$ is not pseudocompact, then the following statements are equivalent:

(i) the family $\left\{C_{K}(X, V)\right\}_{K \in \mathscr{K}(X)}$ satisfies ACP;

(ii) $X \cong \mathbb{N}$ (discrete) and $V$ is finite dimensional.

The proofs of Theorems $\mathrm{A}$ and $\mathrm{B}$ are presented in Section 3 , and are based on basic properties of long colimits established in Section 2 .

Another interesting way to obtain a family of topological groups indexed by the compact subsets of a space $X$ is considering $\operatorname{Homeo}_{K}(X)$, the homeomorphisms that are the identity outside a given compact set $K \in \mathscr{K}(X)$, equipped with the compact open topology. The notion of support, which plays a central role in Theorem $\mathrm{A}$, can also be defined for homeomorphisms: the support of $h: X \rightarrow X$ is $\operatorname{cl}_{X}\{x \in X \mid h(x) \neq x\}$. It is not hard to show that $\left\{\operatorname{Homeo}_{K}(\mathbb{R})\right\}_{K \in \mathscr{K}(\mathbb{R})}$ does not satisfy ACP. In fact, a more general negative result, in the flavour of Theorem $B$, holds.

Theorem C. Let $X$ be a locally compact metrizable space that is not compact, and suppose that $\operatorname{Homeo}_{\bar{U}}(X)$ is not locally compact for every non-empty open set $U$ with $\bar{U}$ compact. Then $\left\{\text { Homeo }_{K}(X)\right\}_{K \in \mathscr{K}(X)}$ does not satisfy ACP.

The proof of Theorem $\mathrm{C}$ is presented in Section 4, where we establish a partial analogue of Theorem $\mathrm{B}$ for homeomorphism groups, and show that a stronger, more general version of Theorem C holds. Notably, the spaces satisfying the conditions of Theorem $\mathrm{C}$ are not long. For certain long spaces, the following analogue to Theorem $\mathrm{A}$ holds.

For a Tychonoff space $X$, we put $\mathrm{Homeo}_{c p t}(X)$ for the set of compactly supported homeomorphisms of $X$. Every compactly supported homeomorphism on $X$ can be extended to a homeomorphism of the Stone-Čech compactification $\beta X$ of $X$ that is the identity on the remainder $\beta X \backslash X$. Thus, the group $\mathrm{Homeo}_{c p t}(X)$ of compactly supported homeomorphisms of $X$ is a subgroup of $\operatorname{Homeo}(\beta X)$, and we may equip $\operatorname{Homeo}_{c p t}(X)$ with the compact-open topology induced by $\beta X$. Then $\operatorname{Homeo}_{K}(X)=\operatorname{Homeo}_{K}(\beta X)$ is a topological subgroup of $\operatorname{Homeo}_{c p t}(X)$ for every $K \in \mathscr{K}(X)$.

Definition 1.2. A Tychonoff space $X$ has the Compactly Supported Homeomorphism Property $(C S H P)$ if

$$
\underset{K \in \mathscr{K}(X)}{\operatorname{colim}} \operatorname{Homeo}_{K}(X)=\text { Homeo }_{c p t}(X),
$$

where the left-hand side is equipped with the colimit space topology.

Clearly, if $X$ has CSHP, then $\left\{\operatorname{Homeo}_{K}(X)\right\}_{K \in \mathscr{K}(X)}$ satisfies ACP; however, the converse is false. For example, if $X$ is an infinite discrete set, then each $\operatorname{Homeo}_{K}(X)$ is finite, and so $\left\{\text { Homeo }_{K}(X)\right\}_{K \in \mathscr{K}(X)}$ satisfies ACP, but $X$ does not have CSHP (see Corollary 5.6).

Theorem D. Suppose that 
(a) $X=\mathbb{L}_{\geq 0}$; or

(b) $X=\mathbb{L}$; or

(c) $X=\kappa^{n} \times \lambda_{1} \times \cdots \times \lambda_{j}$, where $\kappa$ is an uncountable regular cardinal, $\lambda_{1}, \ldots, \lambda_{j}$ are successor ordinals smaller than $\kappa$, and $n, j \in \mathbb{N}$.

Then $X$ has CSHP.

The proof of Theorem $\mathrm{D}$ is presented in Section 5, where we study CSHP.

Finally, we turn to posing some open problems.

The directed limit of $T_{1}$-spaces or normal spaces is again $T_{1}$ or normal, respectively; however, the directed limit of Hausdorff or Tychonoff spaces need not be Hausdorff or Tychonoff ([9]).

Problem I. Let $\left\{G_{\alpha}\right\}_{\alpha \in \mathbb{I}}$ be a long directed family of topological groups with closed embeddings as bonding maps.

(a) Is the colimit group topology $\mathcal{A}$ Hausdorff?

(b) Is the colimit space topology $\mathcal{T}$ Hausdorff?

There is a small gap between the negative statement established in Theorem $B$ and the positive one in Theorem $\mathrm{A}$. There are pseudocompact spaces that are not long ([13]).

Problem II. Let $X$ be a pseudocompact Tychonoff space that is not long. Does $\left\{C_{K}(X, \mathbb{R}\}_{K \in \mathcal{K}(X)}\right.$ satisfy ACP?

There is a big gap between the negative statement established in Theorem $\mathrm{C}$ (or its generalization in Section (4) and the positive one in Theorem D. There are many long locally compact Tychonoff spaces that are not compact, such as finite powers of the long line or the long ray, but it is not known whether they satisfy CSHP.

Problem III. Characterize the long, Tychonoff, non-compact spaces $X$ that satisfy CSHP.

\section{Long families and tightness}

A directed family of topological spaces $\left\{X_{\alpha}\right\}_{\alpha \in \mathbb{I}}$ is called strict if every bonding map $X_{\alpha} \rightarrow X_{\beta}$ is an embedding. A topology $\mathscr{S}$ on the set-theoretic union $X=\bigcup_{\alpha \in \mathbb{I}} X_{\alpha}$ is admissible if it makes each inclusion $X_{\alpha} \rightarrow(X, \mathscr{S})$ an embedding. If there is an admissible topology on $X$, then the family is strict. Furthermore, it can easily be seen that if the bonding maps are closed (or open) embeddings, the colimit space topology

$$
\mathscr{T}:=\left\{U \subseteq X \mid U \cap X_{\alpha} \text { is open in } X_{\alpha} \text { for all } \alpha \in \mathbb{I}\right\}
$$

of a family of spaces is admissible.

In this section, we provide a sufficient condition for the colimit space topology to be the only admissible one (Proposition 2.3), and use it to show that ACP holds for many long families of topological groups.

Recall that the tightness $t(X, \mathscr{S})$ of a topological space $(X, \mathscr{S})$ is the smallest cardinal $\kappa$ such that every point $p$ in the closure of a subset $A \subseteq X$ is in the closure of a subset $C \subseteq A$ with $|C| \leq \kappa$. One says that a space is $\kappa$-tight [countably tight] if $t(X, \mathscr{S}) \leq \kappa$ [if $t(X, \mathscr{S})=\aleph_{0}$ ]. For ease of reference, we start off with a well-known characterization of the tightness. 
Lemma 2.1. Let $X$ be a topological space and $\kappa$ be a cardinal. The following statements are equivalent:

(i) $t(X) \leq \kappa$;

(ii) $X=\operatorname{colim}_{C \leq X,|C| \leq \kappa} C$ in Top, that is, for every topological space $Y$ and $f: X \rightarrow Y$, if $f_{\mid C}$ is continuous for every subset $C \subseteq X$ with $|C| \leq \kappa$, then $f$ is continuous on $X$.

Proof. (i) $\Rightarrow$ (ii): Let $f: X \rightarrow Y$ be a map such that $f_{\mid C}$ is continuous for every subset $C \subseteq X$ with $|C| \leq \kappa$. To show that $f$ is continuous, let $A \subseteq X$ and $x \in \operatorname{cl} A$. Since $t(X) \leq \kappa$, there is $C \subseteq A$ with $|C| \leq \kappa$ such that $x \in \operatorname{cl} C$. Put $D=C \cup\{x\}$. By our assumption, $f_{\mid D}$ is continuous, and so

$$
f(x) \in f\left(\operatorname{cl}_{D} C\right) \subseteq \operatorname{cl} f(C) \subseteq \operatorname{cl} f(A) .
$$

Thus, $f(\operatorname{cl} A) \subseteq \operatorname{cl} f(A)$, as desired.

(ii) $\Rightarrow$ (i): For $A \subseteq X$, put $\mathrm{cl}^{\kappa}(A):=\bigcup\{\operatorname{cl} C|C \subseteq A| C \mid, \leq \kappa\}$. It is easily shown that $\mathrm{cl}^{\kappa}$ is a Kuratowski closure operator, and as such it defines a topology $\mathscr{T}_{\kappa}$ on $X([5,1.2 .7])$. The topology $\mathscr{T}_{\kappa}$ is finer than the original topology $\mathscr{T}$ of $X$, and by (ii), the identity map $(X, \mathscr{T}) \rightarrow\left(X, \mathscr{T}_{\kappa}\right)$ is continuous. Therefore, $\mathscr{T}=\mathscr{T}_{\kappa}$, and hence $\mathrm{cl} A=\mathrm{cl}^{\kappa} A$ for every $A \subseteq X$.

Definition 2.2. Given a cardinal $\kappa$, a directed set $(\mathbb{I}, \leq)$ is $\kappa$-long if every subset $C \subseteq \mathbb{I}$ with $|C| \leq \kappa$ has an upper bound in $\mathbb{I}$. One says that $\mathbb{I}$ is long if it is $\aleph_{0}$-long. A directed family of spaces or groups is $\kappa$-long [long] if it is indexed by a $\kappa$-long [long] set.

A totally ordered set is long if and only if its cofinality is not $\omega$; however, this equivalence fails for ordered sets that are not totally ordered. For example, $\omega \times \omega_{1}$ with the product order has cofinality $\omega_{1}$, but it is not long, because $\omega \times\{0\}$ has no upper bound.

Proposition 2.3. Let $\left\{X_{\alpha}\right\}_{\alpha \in \mathbb{I}}$ be a $\kappa$-long family of topological spaces. For an admissible topology $\mathscr{S}$ on $X=\bigcup_{\alpha \in \mathbb{I}} X_{\alpha}$, the following are equivalent:

(i) $t(X, \mathscr{S}) \leq \kappa$;

(ii) $\mathscr{S}=\mathscr{T}$ (the colimit space topology), and $t\left(X_{\alpha}, \mathscr{S}\right) \leq \kappa$ for all $\alpha \in \mathbb{I}$.

Proof. (i) $\Rightarrow$ (ii): One has $\mathscr{S} \subseteq \mathscr{T}$, because the embeddings $X_{\alpha} \rightarrow(X, \mathscr{S})$ induce a continuous $\operatorname{map}(X, \mathscr{T}) \rightarrow(X, \mathscr{S})$. For the reverse inclusion, we show that $\iota:(X, \mathscr{S}) \rightarrow(X, \mathscr{T})$ is continuous. Let $C \subseteq X$ be such that $|C| \leq \kappa$. Since $\mathbb{I}$ is $\kappa$-long, there is $\alpha \in \mathbb{I}$ such that $C \subseteq X_{\alpha}$. The subspace topology induced by $\mathscr{S}$ on $C$ coincides with the topology induced by $X_{\alpha}$, because $\mathscr{S}$ is admissible. Thus,

$$
\iota_{\mid C}:\left(C, \mathscr{S}_{\mid C}\right) \rightarrow X_{\alpha} \rightarrow(X, \mathscr{T})
$$

is continuous. Therefore, $\iota$ is continuous, because $(X, \mathscr{S})$ is $\kappa$-tight. Hence, $\mathscr{S}=\mathscr{T}$. The second statement follows from $X_{\alpha}$ being a subspace of $(X, \mathscr{S})$.

(ii) $\Rightarrow$ (i): It follows from Lemma 2.1 that the colimit of $\kappa$-tight spaces is $\kappa$-tight.

Corollary 2.4. Let $\left\{G_{\alpha}\right\}_{\alpha \in \mathbb{I}}$ be a $\kappa$-long family of topological groups, and endow $G=\bigcup_{\alpha \in \mathbb{I}} G_{\alpha}$ with the colimit space topology $\mathscr{T}$. If the product $G \times G$ is $\kappa$-tight, then $G$ is a topological group, and $\left\{G_{\alpha}\right\}_{\alpha \in \mathbb{I}}$ satisfies ACP. 
Proof. Put $X_{\alpha}=G_{\alpha} \times G_{\alpha}$ for every $\alpha \in \mathbb{I}$. Since $\mathscr{T} \times \mathscr{T}$ is admissible on $G \times G=\bigcup_{\alpha \in \mathbb{I}} X_{\alpha}$, by Proposition 2.3, $G \times G$ coincides with the colimit of the spaces $\left\{X_{\alpha}\right\}_{\alpha \in \mathbb{I}}$. Therefore, the multiplication $G \times G \rightarrow G$ is continuous.

The following construction provides a wealth of examples of countably tight spaces that need not be first countable. Let $\left\{\left(Y_{j}, 0_{j}\right)\right\}_{j \in J}$ be a family of spaces with base points. The support of $y=\left(y_{j}\right) \in \prod_{j \in J} Y_{j}$ is $\operatorname{supp}(y):=\left\{j \in J \mid y_{j} \neq 0_{j}\right\}$. The $\Sigma$-product of the family is

$$
\sum_{j \in J}\left(Y_{j}, 0_{j}\right):=\left\{y \in \prod_{j \in J} Y_{j} \mid \operatorname{supp}(y) \text { is countable }\right\}
$$

equipped with the topology induced by the product topology.

Theorem 2.5. ([2, 6.16]) Let $\left\{\left(Y_{j}, 0_{j}\right)\right\}_{j \in J}$ be a family of first countable spaces with base points. Then $\sum_{j \in J}\left(Y_{j}, 0_{j}\right)$ is countably tight.

Corollary 2.6. Let $\left\{G_{j}\right\}_{j \in J}$ be a family of metrizable topological groups. Put $\mathbb{I}=[J] \leq \omega$ ordered by inclusion, and for $D \in \mathbb{I}$, put $G_{D}=\prod_{j \in D} G_{j} \times \prod_{j \notin D}\left\{e_{j}\right\}$ with the product topology. Then $\left\{G_{D}\right\}_{D \in \mathbb{I}}$ satisfies $A C P$, and $\operatorname{colim}_{D \in \mathbb{I}} G_{D}=\sum_{j \in J}\left(G_{j}, e_{j}\right)$.

Proof. Put $G:=\bigcup_{D \in \mathbb{I}} G_{D} \subseteq \prod_{j \in J} G_{j}$, and let $\mathscr{S}$ denote the product topology on $G$. By Theorem 2.5, $(G, \mathscr{S})$ is countably tight, and it follows from the construction that $\mathscr{S}$ is admissible. Thus, by Proposition $2.3, \mathscr{S}$ coincides with the colimit space topology $\mathscr{T}$, because the indexing set $\mathbb{I}$ is long. Therefore, $(G, \mathscr{T})$ is a topological group, being a topological subgroup of $\prod_{j \in J} G_{j}$. Hence, $\mathscr{T}$ coincides with the colimit group topology.

Example 2.7. Observe that $\mathbb{I}$ cannot be replaced with the set of finite subsets of $J$ in Corollary 2.6. Let $J=\mathbb{N}$, and let each $G_{j}$ be any metrizable group that is not locally compact. Then $G_{D}$ is metrizable but not locally compact for every non-empty finite $D \subseteq J$ and the bonding maps are not open, because the $G_{j}$ are not discrete. Thus, by Theorem 1.1, the colimit space topology is not a group topology.

Theorem 2.5 has an interesting consequence for permutation groups too. For a set $J$, let $\operatorname{Sym}(J)$ denote the group of permutations of $J$ equipped with the pointwise topology induced by $J^{J}$, where $J$ is equipped with the discrete topology. It is well known that $\operatorname{Sym}(J)$ is a topological group. The support of a permutation $\sigma$ is the set $\operatorname{supp}(\sigma)=\{x \in J \mid \sigma(x) \neq x\}$. Let $\operatorname{Sym}_{\omega}(J)$ denote the subgroup of $\operatorname{Sym}(J)$ consisting of permutations with countable support.

Corollary 2.8. Let $J$ be a set, put $\mathbb{I}=[J] \leq \omega$ ordered by inclusion, and for $D \in \mathbb{I}$, put

$$
G_{D}=\{\sigma \in \operatorname{Sym}(J) \mid \operatorname{supp}(\sigma) \subseteq D\}
$$

equipped with the subgroup topology. Then $\left\{G_{D}\right\}_{D \in \mathbb{I}}$ satisfies $A C P$, and $\underset{D \in \mathbb{I}}{\operatorname{colim}} G_{D}=\operatorname{Sym}_{\omega}(J)$. 
Proof. Consider the family $\{(J, x)\}_{x \in J}$ of spaces with base points. The support of a permutation $\sigma \in \operatorname{Sym}(J)$ coincides with the $\operatorname{supp}(\sigma)$ of $\sigma$ as an element of $J^{J}$. Thus,

$$
\operatorname{Sym}_{\omega}(J)=\operatorname{Sym}(J) \cap\left(\sum_{x \in J}(J, x)\right) .
$$

Therefore, by Theorem 2.5, $\operatorname{Sym}_{\omega}(J)$ is countably tight. It follows from the construction that the topology of $\operatorname{Sym}_{\omega}(J)$ is admissible, and so, by Proposition 2.3, the topology of $\operatorname{Sym}_{\omega}(J)$ coincides with the colimit space topology $\mathscr{T}$, because the indexing set $\mathbb{I}$ is long. Hence, $\left.\operatorname{Sym}_{\omega}(J), \mathscr{T}\right)$ is a topological group, and $\mathscr{T}$ coincides with the colimit group topology.

We turn now to families of metrizable spaces and groups with well-behaved bonding maps.

Theorem 2.9. Let $\left\{\left(X_{\alpha}, d_{\alpha}\right)\right\}_{\alpha \in \mathbb{I}}$ be a strict long family of metrizable spaces. If the bonding maps $\left(X_{\alpha}, d_{\alpha}\right) \rightarrow\left(X_{\beta}, d_{\beta}\right)$ are Lipschitz with a fixed constant $L>0$ (in particular, if they are isometries), then

$$
d(x, y):=\limsup _{\alpha \in \mathbb{I}} d_{\alpha}(x, y)=\inf _{\alpha \in \mathbb{I}} \sup \left\{d_{\beta}(x, y) \mid x, y \in X_{\beta} \text { and } \beta \geq \alpha\right\}
$$

is a metric on $X=\bigcup_{\alpha \in \mathbb{I}} X_{\alpha}$, and it induces the colimit space topology $\mathscr{T}$.

In order to prove Theorem 2.9, we need a technical lemma.

Lemma 2.10. Let $(\mathbb{I}, \leq)$ be a long directed set and $f:(\mathbb{I}, \leq) \rightarrow(\mathbb{R}, \leq)$ a monotone function. Then $f$ is eventually constant, that is, there is $\beta \in \mathbb{I}$ and $s \in \mathbb{R}$ such that $f(\gamma)=$ s for all $\gamma \geq \beta$.

Proof. By replacing $f$ with arctan of or $\arctan \circ(-f)$, we may assume without loss of generality that $f$ is non-decreasing and bounded. Put $s=\sup f(\mathbb{I})$. For every $n \in \mathbb{N} \backslash\{0\}$, there is $\beta_{n} \in \mathbb{I}$ such that $f\left(\beta_{n}\right)>s-\frac{1}{n}$. Since $\mathbb{I}$ is a long directed set, there is $\beta \in \mathbb{I}$ such that $\beta \geq \beta_{n}$ for every $n$. By monotonicity, for every $\gamma \geq \beta$, one has $s \geq f(\gamma) \geq f\left(\beta_{n}\right)>s-\frac{1}{n}$, and thus $f(\gamma)=s$.

Proof of Theorem 2.9 We show that: (1) $d$ is a metric; and (2) $d$ induces an admissible topology on $X$. By Proposition 2.3, it will follow that $d$ induces the colimit space topology on $X$.

For each $\alpha \in \mathbb{I}$ and $x, y \in X_{\alpha}$, put

$$
\widetilde{d}_{\alpha}(x, y)=\sup _{\beta \geq \alpha} d_{\beta}(x, y) .
$$

Then $d_{\alpha} \leq \widetilde{d}_{\alpha} \leq L d_{\alpha}$, and so $\widetilde{d}_{\alpha}$ is a metric on $X_{\alpha}$ that is (strongly) equivalent to $d_{\alpha}$. For $\beta \leq \gamma$ and $x, y \in X_{\beta}$, one has $\widetilde{d}_{\beta}(x, y) \geq \widetilde{d}_{\gamma}(x, y)$. Thus, by Lemma2.10.

$$
d(x, y)=\min _{\beta \in \mathbb{I}}\left\{\widetilde{d}_{\beta}(x, y) \mid x, y \in X_{\beta}\right\}
$$

and for every $x, y \in X$, there is $\beta(x, y) \in \mathbb{I}$ such that $d(x, y)=\widetilde{d}_{\gamma}(x, y)$ for every $\gamma \geq \beta(x, y)$. Since $\mathbb{I}$ is long, for every countable subset $C \subseteq X$ there is $\beta(C) \in \mathbb{I}$ such that $d(x, y)=\widetilde{d}_{\gamma}(x, y)$ for every $x, y \in C$ and $\gamma \geq \beta(C)$. Therefore, $d$ is a metric, and on every countable set $C \subseteq X$, it induces the same topology as $d_{\gamma}$ for $\gamma$ large enough. Hence, $d$ is admissible, because the maps $X_{\alpha} \rightarrow X_{\gamma}$ are embeddings and metric spaces are countably tight. 
Corollary 2.11. Let $\left\{\left(G_{\alpha}, d_{\alpha}\right)\right\}_{\alpha \in \mathbb{I}}$ be a strict long family of metrizable topological groups with bonding maps that are Lipschitz with a fixed constant $L>0$ (in particular, isometries). Then $\left\{G_{\alpha}\right\}_{\alpha \in \mathbb{I}}$ satisfies ACP, and the colimit topology on $G=\bigcup_{\alpha \in \mathbb{I}} G_{\alpha}$ is generated by the metric

$$
d(x, y):=\limsup _{\alpha \in \mathbb{I}} d_{\alpha}(x, y)=\inf _{\alpha \in \mathbb{I}} \sup \left\{d_{\beta}(x, y) \mid x, y \in G_{\beta} \text { and } \beta \geq \alpha\right\} .
$$

Proof. By Theorem 2.9, the colimit space topology $\mathscr{T}$ on $G$ is generated by the metric $d$, and thus $\mathscr{T} \times \mathscr{T}$ is metrizable on $G \times G$; in particular, it is countably tight. Therefore, by Corollary 2.4 , the statement follows.

Example 2.12. The requirement of having a uniform bound for the Lipschitz constants of the bonding cannot be omitted in Theorem 2.9 and Corollary 2.11, Let $M$ be a non-trivial metrizable topological group, fix a metric $d \leq 1$ on $M$, and put $G_{\alpha}:=M^{\alpha}=\prod_{\gamma<\alpha} M$ for $\alpha<\omega_{1}$. Since $\omega_{1}$ is cofinal in $\left[\omega_{1}\right]^{\leq \omega}$, it follows from Corollary 2.6 that $\underset{\alpha<\omega_{1}}{\operatorname{colim}} G_{\alpha}=\sum_{\alpha<\omega_{1}}(M, e)$, and thus the colimit space topology is not metrizable. (In fact, it has an uncountable pseudocharacter, and so it cannot be first countable.)

We construct by transfinite induction a metric $d_{\alpha} \leq 1$ on each $G_{\alpha}$ such that for every $\beta<\alpha$, the embedding $\iota_{\beta \alpha}: G_{\beta} \rightarrow G_{\alpha}$ and the projection $\pi_{\alpha \beta}: G_{\alpha} \rightarrow G_{\beta}$ are Lipschitz. Suppose that such $\left\{d_{\gamma}\right\}_{\gamma<\alpha}$ have already been constructed. If $\alpha=\delta+1$ is a successor ordinal, we put

$$
d_{\alpha}(x, y):=\max \left\{d_{\delta}\left(x_{<\delta}, y_{<\delta}\right), d\left(x_{\delta}, y_{\delta}\right)\right\}
$$

for $x=\left(x_{\gamma}\right)_{\gamma<\alpha}, y=\left(y_{\gamma}\right)_{\gamma<\alpha} \in G_{\alpha}$. The embedding $\iota_{\delta \alpha}$ is an isometry, and $\pi_{\alpha \delta}$ is Lipschitz with constant 1 . Thus, by the inductive hypothesis, the composites $\iota_{\beta \alpha}=\iota_{\beta \delta} \iota_{\delta \alpha}$ and $\pi_{\alpha \beta}=\pi_{\alpha \delta} \pi_{\delta \beta}$ are Lipschitz for every $\beta<\alpha$. If $\alpha$ is a limit ordinal, pick a strictly increasing sequence $\alpha_{i}<\alpha$ such that $\sup \alpha_{i}=\alpha$. For every $i<\omega$, pick $L_{i} \geq 1$ such that $\iota_{\alpha_{n} \alpha_{i}}$ is $L_{i}$-Lipschitz for every $n \leq i$. For $x=\left(x_{\gamma}\right)_{\gamma<\alpha}, y=\left(y_{\gamma}\right)_{\gamma<\alpha} \in G_{\alpha}$, put

$$
d_{\alpha}(x, y):=\sum_{i<\omega} \frac{1}{L_{i} 2^{i+1}} d_{\alpha_{i}}\left(x_{<\alpha_{i}}, y_{<\alpha_{i}}\right)
$$

In what follows, for a Lipschitz map $f$, we put $\operatorname{Lip}(f)$ for the smallest Lipschitz constant for $f$. Let $n<\omega$. The projection $\pi_{\alpha \alpha_{n}}$ is Lipschitz, because $d_{\alpha_{n}}\left(x_{<\alpha_{n}}, y_{<\alpha_{n}}\right) \leq 2^{n+1} L_{n} d_{\alpha}(x, y)$. To see that the embedding $\iota_{\alpha_{n} \alpha}$ is Lipschitz too, suppose that $x, y \in G_{\alpha_{n}} \subseteq G_{\alpha}$. If $i<n$, then one has $d_{\alpha_{i}}\left(x_{<\alpha_{i}}, y_{<\alpha_{i}}\right) \leq \operatorname{Lip}\left(\pi_{\alpha_{n} \alpha_{i}}\right) d_{\alpha_{n}}\left(x_{<\alpha_{n}}, y_{<\alpha_{n}}\right)$. On the other hand, if $n \leq i$, then $\iota_{\alpha_{n} \alpha_{i}}\left(x_{<\alpha_{n}}\right)=x_{<\alpha_{i}}$ and $\iota_{\alpha_{n} \alpha_{i}}\left(y_{<\alpha_{n}}\right)=y_{<\alpha_{i}}$, and so $d_{\alpha_{i}}\left(x_{<\alpha_{i}}, y_{<\alpha_{i}}\right) \leq L_{i} d_{\alpha_{n}}\left(x_{<\alpha_{n}}, y_{<\alpha_{n}}\right)$. Therefore,

$$
\begin{aligned}
d_{\alpha}(x, y) & =\sum_{i<n} \frac{1}{L_{i} 2^{i+1}} d_{\alpha_{i}}\left(x_{<\alpha_{i}}, y_{<\alpha_{i}}\right)+\sum_{i \geq n} \frac{1}{L_{i} 2^{i+1}} d_{\alpha_{i}}\left(x_{<\alpha_{i}}, y_{<\alpha_{i}}\right) \\
& \leq \sum_{i<n} \frac{\operatorname{Lip}\left(\pi_{\alpha_{n} \alpha_{i}}\right)}{L_{i} 2^{i+1}} d_{\alpha_{n}}\left(x_{<\alpha_{n}}, y_{<\alpha_{n}}\right)+\sum_{i \geq n} \frac{1}{2^{i+1}} d_{\alpha_{n}}\left(x_{<\alpha_{n}}, y_{<\alpha_{n}}\right) \\
& \leq\left(\sum_{i<n} \frac{\operatorname{Lip}\left(\pi_{\alpha_{n} \alpha_{i}}\right)}{L_{i} 2^{i+1}}+1\right) d_{\alpha_{n}}\left(x_{<\alpha_{n}}, y_{<\alpha_{n}}\right) .
\end{aligned}
$$


This shows that $\iota_{\alpha_{n} \alpha}$ is Lipschitz. If $\beta<\alpha$, then there is $n<\omega$ such that $\beta \leq \alpha_{n}$, and by the inductive hypothesis, the composites $\iota_{\beta \alpha}=\iota_{\beta \alpha_{n}} \iota_{\alpha_{n} \alpha}$ and $\pi_{\alpha \beta}=\pi_{\alpha \alpha_{n}} \pi_{\alpha_{n} \beta}$ are Lipschitz.

Let $\mathrm{TVS}_{\mathbb{K}}$ denote the category of topological vector spaces over $\mathbb{K} \in\{\mathbb{R}, \mathbb{C}\}$ and their continuous homomorphisms, and let LCTVS $\mathbb{K}$ denote its full subcategory consisting of locally convex spaces.

Corollary 2.13. Let $\left\{E_{\alpha}\right\}_{\alpha \in \mathbb{I}}$ be a long family of Banach spaces over $\mathbb{K}$ (where $\mathbb{K} \in\{\mathbb{R}, \mathbb{C}\}$ ) with isometries as bonding maps. Then:

(a) $\left\{E_{\alpha}\right\}_{\alpha \in \mathbb{I}}$ satisfies ACP;

(b) $E=\bigcup_{\alpha \in \mathbb{I}} E_{\alpha}$ with the colimit space topology $\mathscr{T}$ is a Banach space; and

(c) $(E, \mathscr{T})$ is the colimit of $\left\{E_{\alpha}\right\}_{\alpha \in \mathbb{I}}$ in $\operatorname{TVS}_{\mathbb{K}}$ and $\mathrm{LCTVS}_{\mathbb{K}}$.

Proof. Statement (a) follows from Corollary 2.11, By Theorem 2.9, the topology $\mathscr{T}$ is generated by the metric

$$
d(x, y)=\min _{\alpha \in \mathbb{I}}\left\{d_{\alpha}(x, y) \mid x, y \in E_{\alpha}\right\}=\|x-y\|_{\alpha}, \text { where } x, y \in E_{\alpha}
$$

Thus, the topology $\mathscr{T}$ is generated by the norm

$$
\|x\|=\|x\|_{\alpha}, \text { where } x \in E_{\alpha} .
$$

In particular, $(E, \mathscr{T})$ is a locally convex topological vector space, and (c) follows. In order to show (b), we prove that the normed space $(E,\|\cdot\|)$ is complete. To that end, let $\left\{x_{n}\right\} \subseteq E$ be a Cauchy-sequence. Since $\mathbb{I}$ is long, there is $\alpha \in \mathbb{I}$ such that $x_{n} \in E_{\alpha}$ for every $n$. Thus, $\left\{x_{n}\right\}$ is a Cauchy-sequence in the Banach space $E_{\alpha}$, and $x_{n} \longrightarrow x_{0}$ for $x_{0} \in E_{\alpha}$. Hence, $x_{n} \longrightarrow x_{0}$ in $E$.

Example 2.14. Let $J$ be an uncountable set, put $\mathbb{I}=[J] \leq \omega$, and let $\mathbb{K} \in\{\mathbb{R}, \mathbb{C}\}$. By Corollary 2.13 , for $1 \leq p<\infty$, the space

$$
\ell^{p}(J, \mathbb{K})=\bigcup_{D \in \mathbb{I}} \ell^{p}(D, \mathbb{K})
$$

is the colimit of $\left\{\ell^{p}(D, \mathbb{K})\right\}_{D \in \mathbb{I}}$ in Top, $\operatorname{TVS}_{\mathbb{K}}$, and $\mathrm{LCTVS}_{\mathbb{K}}$.

Example 2.15. Given a strict long family of topological groups, the colimit space topology need not be a group topology. Let $J$ be an uncountable set, and for every finite subset $F \subseteq J$, put $G_{F}:=\mathbb{R}^{F}$ with the Euclidean topology. Put $\mathbb{I}:=[J] \leq \omega$, and for $D \in \mathbb{I}$, put $G_{D}:=\mathbb{R}^{(D)}$ equipped with the colimit space topology induced by $\left\{G_{F}\right\}_{F \in[D]<\omega}$. Since each $G_{D}$ is locally compact, $G_{D}$ is a topological group. (In fact, it can be shown that $G_{D}$ carries the box topology.) One has

$$
\underset{D \in \mathbb{I}}{\operatorname{colim}} G_{D}=\underset{D \in \mathbb{I}}{\operatorname{colim}} \underset{F \in[D]<\omega}{\operatorname{colim}} G_{F}=\underset{F \in[J]<\omega}{\operatorname{colim}} G_{F}
$$

where the colimits are taken in Top. Bisgaard showed that addition is not continuous in the colimit space topology generated by the family $\left\{G_{F}\right\}_{F \in[J]<\omega}([3$, Theorem $])$. Thus, $\left\{G_{D}\right\}_{D \in \mathbb{I}}$ does not satisfy ACP. 


\section{Continuous maps with compact support}

In this section, we prove Theorems $\mathrm{A}$ and $\mathrm{B}$ concerning groups of continuous functions with compact support. We prove Theorem $\mathrm{A}$ by establishing the following more elaborate statement.

Theorem $\mathbf{A}$. Let $X$ be a long space and $M$ be a metrizable group. For $K \in \mathscr{K}(X)$, let $C_{K}(X, M)$ denote the subgroup of $C_{c p t}(X, M)$ consisting of functions with support in $K$. Then the family $\left\{C_{K}(X, M)\right\}_{K \in \mathscr{K}(X)}$ satisfies $A C P$, and

$$
\underset{K \in \mathscr{K}(X)}{\operatorname{colim}} C_{K}(X, M)=C_{c p t}(X, M) .
$$

Furthermore, if $M$ is a Banach space over $\mathbb{K}$ (where $\mathbb{K} \in\{\mathbb{R}, \mathbb{C}\}$ ), then $C_{\text {cpt }}(X, M)$ is a Banach space, and it is the colimit of $\left\{C_{K}(X, M)\right\}_{K \in \mathscr{K}(X)}$ in $\mathrm{TVS}_{\mathbb{K}}$ and $\mathrm{LCTVS}_{\mathbb{K}}$.

ProOf. Let $\rho$ be a metric on $M$. For each $K \in \mathscr{K}(X)$, equip $C_{K}(X, M)$ with the sup-metric $d_{K}$ on $K$, defined by $d_{K}\left(f_{1}, f_{2}\right):=\sup _{x \in K} \rho\left(f_{1}(x), f_{2}(x)\right)$. The bonding maps are isometries, the family $\left\{\left(C_{K}(X, M), d_{K}\right)\right\}_{K \in \mathscr{K}(X)}$ is strict, and since $X$ is a long space, the family is also long. Thus, by Corollary 2.11, the family satisfies ACP, and the colimit topology on $C_{c p t}(X, M)=\bigcup_{K \in \mathscr{K}(X)} C_{K}(X, M)$ is generated by the metric

$$
d\left(f_{1}, f_{2}\right):=\limsup _{K \in \mathscr{K}(X)} d_{K}\left(f_{1}, f_{2}\right)=\limsup _{K \in \mathscr{K}(X)}\left(\sup _{x \in K} \rho\left(f_{1}(x), f_{2}(x)\right)\right)=\sup _{x \in X} \rho\left(f_{1}(x), f_{2}(x)\right) .
$$

The last statement follows by Corollary 2.13.

Examples 3.1. Let $\mathbb{K} \in\{\mathbb{R}, \mathbb{C}\}$.

(a) For an ordinal $\alpha$ of uncountable cofinality, $C_{c p t}(\alpha, \mathbb{K})=\underset{\beta<\alpha}{\operatorname{colim}} C_{[0, \beta]}(\alpha, \mathbb{K})=\underset{\beta<\alpha}{\operatorname{colim}} C([0, \beta], \mathbb{K})$.

(b) $C_{c p t}\left(\mathbb{L}_{\geq 0}, \mathbb{K}\right)=\underset{\beta<\omega_{1}}{\operatorname{colim}} C_{[0, \beta]}\left(\mathbb{L}_{\geq 0}, \mathbb{K}\right)\left(\omega_{1}\right.$ is identified with $\omega_{1} \times\{0\} \subseteq \mathbb{L}_{\geq 0}$ ).

We establish Theorem $B$ by first proving a stronger result using the following observation.

Remark 3.2. Let $\left\{G_{\alpha}\right\}_{\alpha \in \mathbb{I}}$ be a directed family of topological groups that satisfies ACP, and let $H$ be a closed subgroup of $\operatorname{colim}_{\alpha \in \mathbb{I}} G_{\alpha}$. Then $H=\underset{\alpha \in \mathbb{I}}{\operatorname{colim}}\left(G_{\alpha} \cap H\right)$ ([5, Proposition 2.4.15]), and thus $\left\{G_{\alpha} \cap H\right\}_{\alpha \in \mathbb{I}}$ also satisfies ACP.

Theorem B. Let $X$ be a locally compact Hausdorff space and $V$ a non-trivial metrizable topological vector space. If $X$ contains a regular closed $\sigma$-compact non-compact subset, then the following statements are equivalent:

(i) the family $\left\{C_{K}(X, V)\right\}_{K \in \mathscr{K}(X)}$ satisfies $A C P$;

(ii) $X \cong \mathbb{N}$ (discrete) and $V$ is finite dimensional.

Proof. (ii) $\Rightarrow$ (i): Since $X$ is discrete and countable, $\mathscr{K}(X)=[X]^{<\omega}$ is countable. Since $V$ is finite dimensional, the topological vector space $C_{K}(X, V) \cong V^{|K|}$ is finite dimensional, and hence locally compact for every $K \in \mathcal{K}(X)$ (cf. [14, Theorem 3.6]). Thus, $\left\{C_{K}(X, V)\right\}_{K \in \mathscr{K}(X)}$, being a 
countable family of locally compact groups, satisfies ACP ([10, Theorem 4.1] and [8, Propositions 4.7 and 5.4]).

(i) $\Rightarrow$ (ii): Since $\left\{C_{K}(X, V)\right\}_{K \in \mathscr{K}(X)}$ satisfies ACP, by Remark 3.2, for every closed subgroup $H$ of $G:=\underset{K \in \mathscr{K}(X)}{\operatorname{colim}} C_{K}(X, V)$, one has

$$
H=\operatorname{colim}_{K \in \mathscr{K}(X)}\left(C_{K}(X, V) \cap H\right)
$$

and so $\left\{C_{K}(X, V) \cap H\right\}_{K \in \mathscr{K}(X)}$ satisfies ACP. In particular, if $A$ is a closed subspace of $X$ and $H$ is the (closed) subgroup of $G$ consisting of functions whose support is contained in $A$, then $C_{K}(X, V) \cap H=C_{K \cap A}(X, V)$, and thus $\left\{C_{K}(X, V)\right\}_{K \in \mathscr{K}(A)}$ satisfies ACP.

In order to prove that $X$ is discrete, let $U \subseteq X$ be an open subset such that $\operatorname{cl}_{X} U$ is compact. We show that $U$ is finite. Let $A \subseteq X$ be a regular closed $\sigma$-compact non-compact subspace of $X$. Then $A$ contains a countable increasing family $\left\{K_{n}\right\}_{n=1}^{\infty}$ of compact subsets such that $A=\bigcup_{n=1}^{\infty} K_{n}$. Without loss of generality, we may assume that $\mathrm{cl}_{X} U \subseteq K_{1}$. By enlarging the $K_{n}$ if necessary, we may also assume that $K_{n} \subseteq \operatorname{int}_{A} K_{n+1}$ for every $n$. Then $\left\{K_{n}\right\}_{n=1}^{\infty}$ is cofinal in $\mathscr{K}(A)$, that is, every compact subset of $A$ is contained in one of the $K_{n}$. Thus, by (25),

$$
H=\operatorname{colim}_{n} C_{K_{n}}(X, V)
$$

and in particular, addition is continuous in the colimit space topology on the right-hand side. Therefore, since each $C_{K_{n}}(X, V)$ is metrizable, by Theorem 1.1 ,

(a) $C_{K_{n}}(X, V)$ is locally compact for every $n$, or

(b) there is $n$ such that $C_{K_{n}}(X, V)$ is an open subgroup of $C_{K_{m}}(X, V)$ for every $m>n$.

Since $A$ is regular closed and not compact, $\operatorname{int}_{X} A \backslash K_{n}$ is not empty for every $n$. In particular, there is a continuous non-zero $f: X \rightarrow \mathbb{R}$ with compact support $K$ such that $K \subseteq \operatorname{int}_{X} A \backslash K_{n}$. Since $\left\{K_{n}\right\}_{n=1}^{\infty}$ is cofinal in $\mathscr{K}(A)$, there is $m$ such that $K \subseteq K_{m}$. Thus, $C_{K_{n}}(X, \mathbb{R}) \subsetneq C_{K_{m}}(X, \mathbb{R})$, and it follows that $C_{K_{n}}(X, V) \subsetneq C_{K_{m}}(X, V)$, because $V$ is non-trivial. Consequently, $C_{K_{n}}(X, V)$ cannot be an open subgroup of the (path) connected group $C_{K_{m}}(X, V)$.

Therefore, (b) is not possible, and (a) holds: $C_{K_{n}}(X, V)$ is locally compact for every $n$. Since $C_{K_{n}}(X, V)$ is a topological vector space, it is locally compact if and only if it is finite dimensional (cf. [14, Theorem 3.6]). Hence, (a) implies that each $K_{n}$ is finite and $V$ is finite dimensional. In particular, $U \subseteq K_{1}$ is finite, which shows that $X$ is discrete.

Since $X$ contains a non-compact subset $A$, it cannot be finite, and so it remains to show that $X$ is countable. Fix a one-dimensional subspace $W$ of $V$, and let $H$ denote the (closed) subgroup of $G$ consisting of functions with image in $W$. Then $H \cap C_{K}(X, V)=C_{K}(X, W)=\mathbb{R}^{|K|}$ for every $K \in \mathscr{K}(X)$, and by (25), $\left\{\mathbb{R}^{|K|}\right\}_{K \in \mathscr{K}(X)}$ satisfies ACP. Therefore, by Example 2.15, $X$ is countable.

Theorem $\mathrm{B}$ follows from Theorem $\mathrm{B}$ and the following lemma.

Lemma 3.3. If $X$ is a locally compact Hausdorff space and $X$ is not pseudocompact, then $X$ contains a regular closed $\sigma$-compact non-pseudocompact subset. 
Proof. Let $f: X \rightarrow(0, \infty)$ be a continuous unbounded map. Pick $x_{0} \in X$. For every $n>0$, pick $x_{n+1} \in X$ such that $f\left(x_{n+1}\right)>f\left(x_{n}\right)+2$. Since $f$ is continuous, for every $n \in \mathbb{N}$ there is a neighborhood $U_{n}$ of $x_{n}$ such that $\bar{U}_{n}$ is compact and $f\left(U_{n}\right) \subseteq\left(f\left(x_{n}\right)-1, f\left(x_{n}\right)+1\right)$. To show that $\left\{U_{n}\right\}_{n \in \mathbb{N}}$ is locally finite, let $x \in X$, and pick a neighborhood $V$ of $x$ such that $f(V) \subseteq(f(x)-1, f(x)+1)$. It follows from the choice of the $\left\{x_{n}\right\}_{n \in \mathbb{N}}$ that $V$ meets at most two members of the family $\left\{U_{n}\right\}_{n \in \mathbb{N}}$. Thus,

$$
A:=\bigcup_{n \in \mathbb{N}} \overline{U_{n}}=\overline{\bigcup_{n \in \mathbb{N}} U_{n}}
$$

is regular closed $([5,1.1 .11])$ and $\sigma$-compact. The set $A$ is not pseudocompact, because $f_{\mid A}$ is unbounded.

Theorems $\mathrm{B}$ and $\mathrm{B}$ yield the following corollary.

Corollary 3.4. Let $X$ be a non-discrete locally compact Hausdorff space, and let $V$ be a nontrivial metrizable topological vector space. If

(a) $X$ is $\sigma$-compact and non-compact; or

(b) $X$ is not pseudocompact,

then the family $\left\{C_{K}(X, V)\right\}_{K \in \mathscr{K}(X)}$ does not satisfy ACP.

\section{Homeomorphisms with compact support: negative results}

For a locally compact Hausdorff space, the homeomorphism group Homeo $(X)$ of $X$ equipped with the compact-open topology need not be a topological group; however, if $X$ is compact or locally connected, then the compact-open topology is a group topology on $\operatorname{Homeo}(X)$ ([1, Theorem 4]). For a compact subset $K$ of $X$, let $\operatorname{Homeo}_{K}(X)$ denote the group of homeomorphisms of $X$ that fix every point outside $K$ (that is, $h(x)=x$ for all $x \in X \backslash K$ ), and equip it with the compact-open topology. Then $\operatorname{Homeo}_{K}(X)$ is topologically isomorphic to a subgroup of $\operatorname{Homeo}(K)$, where the latter is equipped with the compact-open topology, and as such, $\operatorname{Homeo}_{K}(X)$ is a topological group.

In this section, we consider when the family $\left\{\operatorname{Homeo}_{K}(X)\right\}_{K \in \mathscr{K}(X)}$ satisfies ACP. We start off by proving a partial analogue of Theorem $B$.

Theorem 4.1. Let $X$ be a locally compact Hausdorff space, and let $A \subseteq X$ be a regular closed, $\sigma$-compact, non-compact, metrizable subset. If $\left\{\operatorname{Homeo}_{K}(X)\right\}_{K \in \mathscr{K}(X)}$ satisfies ACP, then at least one of the following holds:

(i) $\mathrm{Homeo}_{M}(X)$ is locally compact for every compact metrizable subset $M \subseteq X$, or

(ii) there is a regular closed compact $K_{0} \subseteq A$ such that:

(1) for all $K \in \mathscr{K}(A)$ with $K_{0} \subseteq K$, the subset $\operatorname{Homeo}_{K_{0}}(X)$ is open in $\operatorname{Homeo}_{K}(X)$; and

(2) for all $C \in \mathscr{K}\left(\overline{A \backslash K_{0}}\right)$, the group $\operatorname{Homeo}_{C}(X)$ is discrete. 
Proof. We show that if (i) fails, then (ii) holds. To that end, let $M_{0}$ be a compact metrizable subset of $X$ such that $\operatorname{Homeo}_{M_{0}}(X)$ is not locally compact. By replacing $M_{0}$ with $\operatorname{cl}\left(\operatorname{int} M_{0}\right)$ if necessary, we may assume that $M_{0}$ is regular closed. Furthermore, by replacing $A$ with $A \cup M_{0}$ if necessary, we may assume that $M_{0} \subseteq A$.

Since $A$ is $\sigma$-compact, it contains a countable increasing family $\left\{K_{n}\right\}_{n=1}^{\infty}$ of compact subsets such that $A=\bigcup_{n=1}^{\infty} K_{n}$. By enlarging the $K_{n}$ if necessary, we may assume that $M_{0} \subseteq K_{n} \subseteq \operatorname{int}_{A} K_{n+1}$ for every $n$. Then $\left\{K_{n}\right\}_{n=1}^{\infty}$ is cofinal in $\mathscr{K}(A)$, that is, every compact subset of $A$ is contained in one of the $K_{n}$.

Since $\left\{\text { Homeo }_{K}(X)\right\}_{K \in \mathscr{K}(X)}$ satisfies ACP, by Remark 3.2, for every closed subgroup $H$ of $G:=\underset{K \in \mathscr{K}(X)}{\operatorname{colim}} \operatorname{Homeo}_{K}(X)$, one has

$$
H=\underset{K \in \mathscr{K}(X)}{\operatorname{colim}}\left(\operatorname{Homeo}_{K}(X) \cap H\right)
$$

and so $\left\{\text { Homeo }_{K}(X) \cap H\right\}_{K \in \mathscr{K}(X)}$ satisfies ACP. In particular, if $A$ is a closed subspace of $X$ and $H$ is the (closed) subgroup of $G$ consisting of homeomorphisms whose support is contained in $A$, then $\operatorname{Homeo}_{K}(X) \cap H=\operatorname{Homeo}_{K \cap A}(X)$, and thus $\left\{\operatorname{Homeo}_{K}(X)\right\}_{K \in \mathscr{K}(A)}$ satisfies ACP. By (28),

$$
H=\operatorname{colim}_{n} \operatorname{Homeo}_{K_{n}}(X)
$$

and in particular, the group multiplication is continuous in the colimit space topology on the righthand side. Therefore, since each $\operatorname{Homeo}_{K_{n}}(X)$ is metrizable, by Theorem 1.1 ,

(a) $\mathrm{Homeo}_{K_{n}}(X)$ is locally compact for every $n$, or

(b) there is $n_{0}$ such that $\operatorname{Homeo}_{K_{n}}(X)$ is an open subgroup of $\operatorname{Homeo}_{K_{m}}(X)$ for every $m>n_{0}$.

By our assumption, Homeo $M_{0}(X)$ is not locally compact, and $M_{0} \subseteq K_{n}$ for every $n$. So, (a) is not possible, and (b) holds. Put $K_{0}:=\mathrm{cl}_{X}\left(\operatorname{int}_{X} K_{n_{0}}\right)$. Observe that $\operatorname{Homeo}_{K_{0}}(X)=\operatorname{Homeo}_{K_{n_{0}}}(X)$. We show that (ii) holds for $K_{0}$.

To show (ii)(1), let $K \in \mathscr{K}(A)$ such that $K_{0} \subseteq K$. Since $\left\{K_{n}\right\}_{n=1}^{\infty}$ is cofinal in $\mathscr{K}(A)$, there is $m>n_{0}$ such that $K \subseteq K_{m}$. Thus, $\operatorname{Homeo}_{K_{0}}(X)$ is open in $\operatorname{Homeo}_{K_{m}}(X)$, and in particular, Homeo $_{K_{0}}(X)$ is open in $\operatorname{Homeo}_{K}(X)$.

To show (ii)(2), let $C \in \mathscr{K}\left(\overline{A \backslash K_{0}}\right)$, and put $L:=K_{0} \cup C$. Since $\operatorname{int}_{X} K_{0}$ and int ${ }_{X} C$ are disjoint, Homeo $_{K_{0}}(X)$ commutes with $\operatorname{Homeo}_{C}(X)$, and the multiplication map

$$
\operatorname{Homeo}_{K_{0}}(X) \times \operatorname{Homeo}_{C}(X) \rightarrow \operatorname{Homeo}_{L}(X)
$$

is an embedding of topological groups. By (ii)(1), $\operatorname{Homeo}_{K_{0}}(X)$ is open in $\operatorname{Homeo}_{L}(X)$. Thus, Homeo $_{K_{0}}(X)$ is open in $\mathrm{Homeo}_{K_{0}}(X) \times \operatorname{Homeo}_{C}(X)$, and therefore $\operatorname{Homeo}_{C}(X)$ is discrete.

We establish Theorem $\mathrm{C}$ by proving a stronger result.

Theorem C. Let $X$ be a locally compact Hausdorff space that is not compact. Each of the following statements imply the subsequent ones:

(i) $X$ is metrizable, locally Euclidean, and has no isolated points; 
(ii) $X$ is metrizable and $\operatorname{Homeo}_{\bar{U}}(X)$ is not locally compact for every non-empty open set $U$ such that $\bar{U}$ is compact;

(iii) $X$ is normal, locally metrizable, and contains

(1) a compact subset $M_{0}$ such that Homeo $_{M_{0}}(X)$ is not locally compact; and

(2) an infinite discrete closed set $T$ such that for every $t \in T$ and every neighborhood $V$ of $t$ with compact closure, $\mathrm{Homeo}_{\bar{V}}(X)$ is not discrete;

(iv) $X$ contains

(1) a compact metrizable subset $M_{0}$ such that $\operatorname{Homeo}_{M_{0}}(X)$ is not locally compact; and

(2) a regular closed, $\sigma$-compact, and metrizable subset $A$ whose interior contains an infinite discrete closed set $T$ such that for every $t \in T$ and every neighborhood $V$ oft with compact closure, $\operatorname{Homeo}_{\bar{V}}(X)$ is not discrete;

(v) $\left\{\operatorname{Homeo}_{K}(X)\right\}_{K \in \mathscr{K}(X)}$ does not satisfy ACP.

Proof. (i) $\Rightarrow$ (ii): Let $U$ be a non-empty open set whose closure in $X$ is compact. Since $X$ is locally Euclidean, $U$ contains a non-empty open set $V$ such that there is a homeomorphism $h: \mathbb{R}^{n} \rightarrow V$ for some $n \in \mathbb{N}$. Put $W:=h\left((-1,1)^{n}\right)$. Observe that $\bar{W}=h\left([-1,1]^{n}\right)$ and one has Homeo $_{\bar{W}}(X) \cong$ Homeo $_{[-1,1]^{n}}\left(\mathbb{R}^{n}\right)$. The group Homeo $[-1,1]^{n}\left(\mathbb{R}^{n}\right)$ is not locally compact, because it contains a copy of Homeo ${ }_{[-1,1]}(\mathbb{R})$ as a closed subgroup, and the latter is known to be nonlocally compact (it is not even complete with respect to its left uniformity; see, for example [4]). Since $\mathrm{Homeo}_{\bar{W}}(X) \subseteq \mathrm{Homeo}_{\bar{U}}(X)$, it follows that $\mathrm{Homeo}_{\bar{U}}(X)$ is not locally compact.

(ii) $\Rightarrow$ (iii): Since $X$ is metrizable but not compact, it contains an infinite closed discrete set $T$.

(iii) $\Rightarrow$ (iv): Since every compact subset of a locally metrizable space is metrizable, (iii)(1) implies (iv)(1). Without loss of generality, we may assume that $T=\left\{t_{0}, t_{1}, \ldots\right\}$ is countably infinite. In order to show (iv)(2), we construct the set $A$ around $T$.

Let $\left\{U_{i}\right\}_{i \in \mathbb{N}}$ be a family of open sets in $X$ such that $t_{i} \in U_{i}$ and $\bar{U}_{i}$ is compact for every $i \in \mathbb{N}$, and $\bar{U}_{i} \cap \bar{U}_{j}=\emptyset$ for $i \neq j$. (Such a family exists, because $T$ is discrete and countable.) Put $F:=X \backslash \bigcup_{i \in \mathbb{N}} U_{i}$.

Since $F$ and $T$ are closed disjoint sets in the normal space $X$, there are disjoint open sets $U$ and $V$ such that $T \subseteq U$ and $F \subseteq V$. Put $W_{i}:=U_{i} \cap U$. Then $t_{i} \in W_{i}$ and $\overline{W_{i}}$ is compact, and $\left\{W_{i}\right\}_{i \in \mathbb{N}}$ is locally finite. Thus,

$$
A=\bigcup_{i \in \mathbb{N}} \overline{W_{i}}=\overline{\bigcup_{i \in \mathbb{N}} W_{i}}
$$

is regular closed ([5, 1.1.11]), $\sigma$-compact, and its interior contains $T$. Finally, $A$ is metrizable, because it is locally metrizable and Lindelöf.

(iv) $\Rightarrow(v)$ : Assume that $\left\{\operatorname{Homeo}_{K}(X)\right\}_{K \in \mathscr{K}(X)}$ satisfies ACP. Then, by Theorem 4.1, either Homeo $_{M_{0}}(X)$ is locally compact, or $A$ contains a (regular) closed compact subset $K_{0}$ such that Homeo $_{C}(X)$ is discrete for every $C \in \mathscr{K}\left(\overline{A \backslash K_{0}}\right)$. By (iv)(1), the first case is not possible, so suppose that the second case holds. Since $T$ is infinite closed and discrete, $T \nsubseteq K_{0}$. Thus, there is $t_{0} \in T$ such that $t_{0} \notin K_{0}$. Let $V$ be a neighborhood of $t_{0}$ such that $V \subseteq A \backslash K_{0}$ and $\mathrm{cl}_{X} V$ is compact, and put $C:=\mathrm{cl}_{X} V$. Then, by (iv)(2), $\operatorname{Homeo}_{C}(X)$ is not discrete. This contradiction shows that $\left\{\text { Homeo }_{K}(X)\right\}_{K \in \mathscr{K}(X)}$ does not satisfy ACP. 


\section{The Compactly Supported Homeomorphism Property (CSHP)}

Our goal in this section is to prove Theorem $\mathrm{D}$,

Theorem D. Suppose that

(a) $X=\mathbb{L}_{\geq 0}$; or

(b) $X=\mathbb{L} ;$; or

(c) $X=\kappa^{n} \times \lambda_{1} \times \cdots \times \lambda_{j}$, where $\kappa$ is an uncountable regular cardinal, $\lambda_{1}, \ldots, \lambda_{j}$ are successor ordinals smaller than $\kappa$, and $n, j \in \mathbb{N}$.

Then $X$ has CSHP.

We prove parts (a) and (b) of Theorem D by showing that the compact-open topology on $\mathrm{Homeo}_{c p t}(\mathbb{L})$ is countably tight and coincides with the compact-open topology induced by $\beta X$, and then applying Proposition 2.3 .

Proposition 5.1. Let $X=\mathbb{L}_{\geq 0}$ or $X=\mathbb{L}$. Then $\operatorname{Homeo}_{\text {cpt }}(X)$ equipped with the compact-open topology is countably tight.

Pro0f. The statement for $X=\mathbb{L}_{\geq 0}$ follows from the statement for the case $X=\mathbb{L}$, because Homeo $_{c p t}\left(\mathbb{L}_{\geq 0}\right)$ equipped with the compact-open topology is a subspace of $\operatorname{Homeo}_{c p t}(\mathbb{L})$ equipped with the compact-open topology.

Let $X=\mathbb{L}, A \subseteq$ Homeo $_{\text {cpt }}(\mathbb{L})$, and suppose that $f_{0} \in \mathrm{cl} A$. We construct a countable subset $C \subseteq A$ such that $f_{0} \in \operatorname{cl} C$. Since composition is continuous in the compact-open topology, without loss of generality, we may assume that $f_{0}=\mathrm{id}_{\mathbb{L}}$.

For $\alpha<\omega_{1}$, put $K_{\alpha}=[-(\alpha, 0),(\alpha, 0)]$ and $U_{\alpha}=(-(\alpha+1,0),(\alpha+1,0))$. The set

$$
W_{\alpha}:=\left\{h \in \text { Homeo }_{\text {cpt }}(\mathbb{L}) \mid h\left(K_{\alpha}\right) \subseteq U_{\alpha}\right\}
$$

is an open neighborhood of $\mathrm{id}_{\mathbb{L}}$ in the compact open topology, and thus $\operatorname{id}_{\mathbb{L}} \in \operatorname{cl}\left(W_{\alpha} \cap A\right)$. Since $K_{\alpha}$ is compact and $U_{\alpha}$ is metrizable, the space $\mathscr{C}\left(K_{\alpha}, U_{\alpha}\right)$ is metrizable in the compact-open topology. Consequently, there is a sequence $\left\{f_{n}^{(\alpha)}\right\} \subseteq W_{\alpha} \cap A$ such that $f_{n \mid K_{\alpha}}^{(\alpha)} \longrightarrow \operatorname{id}_{K_{\alpha}}$ uniformly.

We construct now an increasing sequence of ordinals $\left\{\alpha_{m}\right\} \subseteq \omega_{1}$ such that supp $f_{n}^{\left(\alpha_{m}\right)} \subseteq K_{\alpha_{m+1}}$ for every $n, m \in \mathbb{N}$. Pick an arbitrary $\alpha_{0}<\omega_{1}$. Suppose that $\alpha_{m}$ has already been constructed. Since $\mathbb{L}$ is a long space, the countable family of compact subsets $\left\{\operatorname{supp} f_{n}^{\left(\alpha_{m}\right)}\right\}_{n \in \mathbb{N}}$ has an upper bound; in particular, there is $\beta<\omega_{1}$ such that $\operatorname{supp} f_{n}^{\left(\alpha_{m}\right)} \subseteq K_{\beta}$ for every $n \in \mathbb{N}$. Put $\alpha_{m+1}:=\max \left(\beta, \alpha_{m}+1\right)$.

Since $\left(\omega_{1}, \leq\right)$ is long, $\gamma:=\sup _{m \in \mathbb{N}} \alpha_{m}$ exists in $\omega_{1}$, and $\operatorname{supp} f_{n}^{\left(\alpha_{m}\right)} \subseteq K_{\gamma}$ for every $n, m \in \mathbb{N}$. Put

$$
C:=\left\{f_{n}^{\left(\alpha_{m}\right)} \mid n, m \in \mathbb{N}\right\} \subseteq A
$$

We show that $\operatorname{id}_{\mathbb{L}} \in \operatorname{cl} C$. Observe that $C \subseteq \operatorname{Homeo}_{K_{\gamma}}(\mathbb{L})$. Pick an order-preserving homeomor$\operatorname{phism} \varphi: K_{\gamma} \rightarrow[-1,1]$. It suffices to show that $\operatorname{id}_{[-1,1]} \in \operatorname{cl}\left(\varphi C \varphi^{-1}\right)$ in $\operatorname{Homeo}([-1,1])$.

Let $\varepsilon>0$. There is $m \in \mathbb{N}$ such that $a:=\varphi\left(-\left(\alpha_{m}, 0\right)\right)<-1+\frac{\varepsilon}{2}$ and $b:=\varphi\left(\alpha_{m}, 0\right)>1-\frac{\varepsilon}{2}$. By our construction, $f_{n \mid K_{\alpha_{m}}}^{\left(\alpha_{m}\right)} \longrightarrow \mathrm{id}_{K_{\alpha_{m}}}$ uniformly, and so $\varphi f_{n \mid K_{\alpha_{m}}}^{\left(\alpha_{m}\right)} \varphi^{-1} \longrightarrow \mathrm{id}_{[a, b]}$ uniformly. Thus, there 
is $n \in \mathbb{N}$ such that $\left|\varphi f_{n \mid K_{\alpha_{m}}}^{\left(\alpha_{m}\right)} \varphi^{-1}(x)-x\right|<\frac{\varepsilon}{2}$ for every $x \in[a, b]$. Since every compactly supported homeomorphism of $\mathbb{L}$ is order-preserving, for every $x \in[b, 1]$, one has

$$
1 \geq \varphi f_{n \mid K_{\gamma}}^{\left(\alpha_{m}\right)} \varphi^{-1}(x) \geq \varphi f_{n \mid K_{\gamma}}^{\left(\alpha_{m}\right)} \varphi^{-1}(b)>b-\frac{\varepsilon}{2}>1-\varepsilon
$$

and similarly, for $x \in[-1, a]$, one has $-1 \leq f_{n \mid K_{\gamma}}^{\left(\alpha_{m}\right)} \varphi^{-1}(x)<-1+\varepsilon$. Hence, $\left|\varphi f_{n \mid K_{\alpha_{m}}}^{(\gamma)} \varphi^{-1}(x)-x\right|<\varepsilon$ for every $x \in[-1,1]$.

Proof of Theorem $\mathbb{D}(\mathbf{A})$-(B). Let $X=\mathbb{L}_{\geq 0}$ or $X=\mathbb{L}$, and let $\alpha X$ denote its one-point compactification. Since $X$ is locally connected, the compact-open topology on $\operatorname{Homeo}(X)$, and thus on Homeo $_{c p t}(X)$, coincides with the compact-open topology induced by $\alpha X$ ([1, Theorems 1 and 4]). The compact-open topology is admissible with respect to the family $\left\{\operatorname{Homeo}_{K}(X)\right\}_{K \in \mathscr{K}(X)}$, and by Proposition 5.1, Homeo ${ }_{c p t}(X)$ is countably tight in the compact-open topology. Thus, by Proposition 2.3,

$$
\underset{K \in \mathscr{K}(X)}{\operatorname{colim}} \operatorname{Homeo}_{K}(X)=\text { Homeo }_{c p t}(X)
$$

where the left-hand side is equipped with the colimit space topology and the right-hand side is equipped with the compact-open topology. Since $\operatorname{Homeo}_{c p t}(X)$ is a topological group, this shows that the family $\left\{\operatorname{Homeo}_{K}(X)\right\}_{K \in \mathscr{K}(X)}$ satisfies ACP. Therefore, it remains to show that the compact-open topology on $\mathrm{Homeo}_{c p t}(X)$ coincides with the compact-open topology induced by $\beta X$.

(a) Since $\beta \mathbb{L}_{\geq 0}=\alpha \mathbb{L}_{\geq 0}([6,16 \mathrm{H} 6])$, the statement follows for $X=\mathbb{L}_{\geq 0}$.

(b) It is easily seen that $\beta \mathbb{L}=\mathbb{L} \cup\{-\infty, \infty\}$. (Since $\mathbb{L}$ is a pushout of two copies of $\mathbb{L}_{\geq 0}$ over $\{0\}$ and $\beta$ preserves pushouts, $\beta \mathbb{L}$ is the pushout of two copies of $\beta \mathbb{L}_{\geq 0}=\alpha \mathbb{L}_{\geq 0}$ over $\{0\}$.) Since $\mathbb{L}$ is open in $\beta \mathbb{L}$, the compact-open topology on $\mathrm{Homeo}_{\text {cpt }}(\mathbb{L})$ (induced by $\mathbb{L}$ ) is coarser than the compact-open topology induced by $\beta X$. In order to prove the converse, let $K \subseteq U \subseteq \beta \mathbb{L}$, where $K$ is compact and $U$ is open, and consider the subbasic neighborhood of the identity

$$
W=\left\{h \in \operatorname{Homeo}_{c p t}(\mathbb{L}) \mid h^{\beta}(K) \subseteq U\right\}
$$

where $h^{\beta}: \beta \mathbb{L} \rightarrow \beta \mathbb{L}$ is the extension of $h$. Since $K$ is compact, it may be covered by finitely many connected closed subsets $I_{1}, \ldots, I_{n}$ of $\beta \mathbb{L}$ such that $I_{j} \subseteq U$. For $j=1, \ldots, n$, put

$$
W_{j}:=\left\{h \in \operatorname{Homeo}_{c p t}(\mathbb{L}) \mid h^{\beta}\left(I_{j}\right) \subseteq U\right\} .
$$

One has $\bigcap_{j=1}^{n} W_{j} \subseteq W$, and so without loss of generality we may assume that $K$ is connected. We distinguish among three cases.

Case 1. If $\{-\infty, \infty\} \subseteq K$, then $K=U=\beta \mathbb{L}$, and thus $W=\mathrm{Homeo}_{c p t}(\mathbb{L})$ is open.

Case 2. If $K \subseteq \mathbb{L}$, then by replacing $U$ with $U \cap \mathbb{L}$, one sees that $W$ is open in the compactopen topology. (Compactly supported homeomorphisms of $\mathbb{L}$ preserve the order, and thus their extensions to $\beta \mathbb{L}$ fix $\infty$ and $-\infty$.) 
Case 3. If $|K \cap\{-\infty, \infty\}|=1$, then without loss of generality we may assume that $\infty \in K$ and $-\infty \notin K$. There are closed connected sets $K_{1}, K_{2} \subseteq K$ such that $K=K_{1} \cup K_{2}, K_{1} \subseteq \mathbb{L}_{\geq 0} \backslash\{0\}$, and $\infty \notin K_{2}$. Put $U_{1}:=U \cap \mathbb{L}_{\geq 0} \backslash\{0\}$ and $U_{2}=U$, and for $i=1,2$, put

$$
W_{i}^{\prime}:=\left\{h \in \text { Homeo }_{\text {cpt }}(\mathbb{L}) \mid h^{\beta}\left(K_{i}\right) \subseteq U_{i}\right\}
$$

One has $W_{1}^{\prime} \cap W_{2}^{\prime} \subseteq W$. By Case $2, W_{2}^{\prime}$ is open in the compact-open topology. Thus, it remains to show that $W_{1}^{\prime}$ is a neighborhood of the identity in the compact-open topology. Put $K^{\prime}:=K_{1} \cup\left(-K_{1}\right)$ and $U^{\prime}=U_{1} \cup\left(-U_{1}\right)$, where - stands for the involution interchanging the two copies of $\alpha \mathbb{L}_{\geq 0}$ in $\beta \mathbb{L}$, and put

$$
W^{\prime}:=\left\{h \in \operatorname{Homeo}_{c p t}(\mathbb{L}) \mid h^{\beta}\left(K^{\prime}\right) \subseteq U^{\prime}\right\}
$$

If $h \in W^{\prime}$, then $h^{\beta}\left(K_{1}\right) \subseteq U_{1} \cup\left(-U_{1}\right)$, and the latter union is disjoint, because $U_{1} \subseteq \mathbb{L}_{\geq 0} \backslash\{0\}$. Thus, the connected set $h^{\beta}\left(K_{1}\right)$ is contained in $U_{1}$ in its entirety, because $h^{\beta}(\infty) \in h^{\beta}\left(K_{1}\right) \in U_{1}$. Therefore, $h \in W^{\prime}$. This shows that $W^{\prime} \subseteq W_{1}^{\prime}$. For $V=\beta \mathbb{L} \backslash K$ and $C=\beta \mathbb{L} \backslash U$, one has

$$
W^{\prime}=\left\{h \in \text { Homeo }_{c p t}(\mathbb{L}) \mid h^{\beta}(V) \supseteq C\right\}=\left\{h^{-1} \in \text { Homeo }_{c p t}(\mathbb{L}) \mid h^{\beta}(C) \subseteq V\right\} .
$$

Since $C, V \subseteq \mathbb{L}$ and the compact-open topology on $\mathrm{Homeo}_{\text {cpt }}(\mathbb{L})$ is a group topology, this proves that $W^{\prime}$ is open in the compact-open topology.

Similarly to the proofs found in Section 2 and the proof of Theorem $\mathrm{A}$, the proofs of parts (a) and (b) of Theorem $\mathrm{D}$ rely on the tightness of the groups involved. Unfortunately, this approach cannot be used to prove part (c) of Theorem D, because $\operatorname{Homeo}_{K}(X)$ need not be countably tight for a big space $X$.

Example 5.2. Let $\kappa$ be an ordinal, and $\lambda<\kappa$ a limit ordinal of uncountable cofinality (for example, $\lambda=\omega_{1}$ and $\left.\kappa=\omega_{2}\right)$. The set $[0, \lambda]=[0, \lambda+1)$ is clopen in $X=\kappa$, and thus

$$
\operatorname{Homeo}_{[0, \lambda]}(\kappa)=\operatorname{Homeo}([0, \lambda]),
$$

where the latter is equipped with the compact-open topology. We show that

$$
t(\operatorname{Homeo}([0, \lambda])) \geq \operatorname{cf}(\lambda) .
$$

Consider the subset $A:=\bigcup_{\beta<\lambda} \operatorname{Homeo}_{[0, \beta]}([0, \lambda])$ of Homeo $([0, \lambda])$. If $B \subseteq A$ such that $|B|<\operatorname{cf}(\lambda)$, then $\tau:=\sup \bigcup_{f \in B} \operatorname{supp}(f)<\lambda$, and thus $B \subseteq$ Homeo $_{[0, \tau]}([0, \lambda])$; in particular, $\bar{B} \subseteq A$. On the other hand, for $h:[0, \lambda] \rightarrow[0, \lambda]$ defined by $h(\alpha+1)=h(\alpha+2)$ and $h(\alpha+2)=\alpha+1$ for limit ordinals $\alpha$ and $h(x)=x$ otherwise, one can easily see that $h \notin A$ but $h \in \bar{A}$. (For every limit ordinal $\beta<\lambda$, put $h_{\beta}(x)=h(x)$ if $x \leq \beta$ and $h_{\beta}(x)=x$ if $x>\beta$. Clearly, $h_{\beta} \in A$, and $h_{\beta} \rightarrow h$ in the uniform topology, which coincides with the compact-open topology.)

The proof of part (c) of Theorem $\mathrm{D}$ consists of a number of steps. We first show that CSHP is inherited by clopen subsets, and reduce the proof to the special case of $X=\kappa^{n}$. We then provide a description of the topology of $\operatorname{Homeo}(K)$ for a compact zero-dimensional space $K$, and use it to describe open neighborhoods of the identity in $\operatorname{Homeo}_{K}\left(\kappa^{n}\right)$. 
Lemma 5.3. Let $X$ be a Tychonoff space and $A \subseteq X$ a clopen subset.

(a) $\mathrm{Homeo}_{c p t}(A)$ naturally embeds as a closed topological subgroup into $\operatorname{Homeo}_{\text {cpt }}(X)$; and

(b) if $X$ has CSHP, then so does $A$.

Proof. (a) Since $A$ is clopen, $\beta A$ embeds as a clopen subset into $\beta X$, and every homeomorphism of $\beta A$ can be extended to a homeomorphism of $\beta X$ by defining it as the identity on $\beta X \backslash \beta A$. Thus, $\operatorname{Homeo}(\beta A)$ embeds as a topological subgroup into $\operatorname{Homeo}(\beta X)$, where the groups are equipped with the respective compact-open topologies. Therefore, $\operatorname{Homeo}_{c p t}(A)$ embeds as a topo- $^{-}$ logical subgroup into $\mathrm{Homeo}_{c p t}(X)$, and one can identify

$$
\text { Homeo }_{c p t}(A)=\left\{f \in \text { Homeo }_{c p t}(X) \mid f_{\mid X \backslash A}=\mathrm{id}_{X \backslash A}\right\} .
$$

Hence, $\mathrm{Homeo}_{c p t}(A)$ is closed in $\mathrm{Homeo}_{c p t}(X)$.

(b) Since $X$ has CSHP, by Remark 3.2, for every closed subgroup $H$ of $\operatorname{Homeo}_{c p t}(X)$, one has

$$
H=\underset{K \in \mathscr{K}(X)}{\operatorname{colim}}\left(\operatorname{Homeo}_{K}(X) \cap H\right) .
$$

In particular, for $H=$ Homeo $_{c p t}(A)$, one obtains

$$
\text { Homeo }_{c p t}(A)=\underset{K \in \mathscr{K}(X)}{\operatorname{colim}}\left(\operatorname{Homeo}_{K}(X) \cap \operatorname{Homeo}_{c p t}(A)\right)=\underset{K \in \mathscr{K}(A)}{\operatorname{colim}} \operatorname{Homeo}_{K}(A),
$$

and hence $A$ has CSHP.

If $\kappa$ is a cardinal and $\lambda_{1}, \ldots, \lambda_{j}<\kappa$ are successor ordinals, then $\kappa^{n} \times \lambda_{1} \times \cdots \times \lambda_{j}$ is a clopen subset of $\kappa^{n+j}$. Thus, Lemma 5.3 yields the following reduction.

Corollary 5.4. Let $\kappa$ be a cardinal. If the space $\kappa^{n}$ has CSHP for every $n$, then $\kappa^{n} \times \lambda_{1} \times \cdots \times \lambda_{j}$ has CSHP for every $n$ and successor ordinals $\lambda_{1}, \ldots, \lambda_{j}<\kappa$.

For a Tychonoff space $X$ and a finite clopen partition $\mathcal{A}=\left\{A_{1}, \ldots, A_{k}\right\}$ of $X$ (i.e., each $A_{i}$ is clopen), put

$$
U(\mathcal{A}):=\left\{f \in \text { Homeo }_{c p t}(X) \mid f\left(A_{i}\right)=A_{i} \text { for all } i\right\}
$$

the subgroup of homeomorphisms $f$ such that each $A_{i}$ is $f$-invariant.

Lemma 5.5. Let $X$ be a zero-dimensional space. Then the family

$$
\{U(\mathcal{A}) \mid \mathcal{A} \text { is a finite clopen partition of } X\}
$$

is a base at the identity for $\mathrm{Homeo}_{c p t}(X)$.

Proof. Since there is a one-to-one correspondence between clopen partitions of $X$ and of $\beta X$, and $\operatorname{Homeo}_{c p t}(X)$ is a topological subgroup of $\operatorname{Homeo}(\beta X)$ (where the latter is equipped with the compact-open topology), we may assume without loss of generality that $X$ is compact. 
If $\mathcal{A}=\left\{A_{1}, \ldots, A_{k}\right\}$ is a finite clopen partition of $X$, then each $A_{i}$ is compact, and

$$
U(\mathcal{A})=\bigcap_{i=1}^{k}\left\{f \in \text { Homeo }_{c p t}(X) \mid f\left(A_{i}\right) \subseteq A_{i}\right\}
$$

is an open neighborhood of the identity in $\operatorname{Homeo}_{c p t}(X)$. Conversely, consider a subbasic open set $W=\left\{f \in\right.$ Homeo $\left._{\text {cpt }}(X) \mid f(K) \subseteq U\right\}$ containing the identity, where $K$ is compact and $U$ is open in $X$. Since $W$ contains the identity, $K \subseteq U$, and there is a clopen subset $A_{1}$ such that $K \subseteq A_{1} \subseteq U$, because $X$ is zero-dimensional. Thus, for $\mathcal{A}:=\left\{A_{1}, X \backslash A_{1}\right\}$, one has $U(\mathcal{A}) \subseteq W$. Since the collection of sets of the form $U(\mathcal{A})$ is a filter base, this completes the proof.

Corollary 5.6. If a Tychonoff space $X$ contains an infinite discrete clopen subset, then $X$ does not have CSHP.

Proof. By Lemma 5.3 (b), it suffices to show that if $X$ is an infinite discrete space, then it does not have CSHP. If $X$ is infinite discrete, then $\operatorname{Homeo}_{K}(X)$ is finite for every $K \in \mathscr{K}(X)$, and thus $\underset{K \in \mathscr{K}(X)}{\operatorname{colim}} \operatorname{Homeo}_{K}(X)$ is discrete too; however, by Lemma 5.5. $\operatorname{Homeo}_{c p t}(X)$ is not discrete, because $U(\mathcal{A})$ is an infinite group for every finite partition $\mathcal{A}$ of $X$.

Our next step is to describe open neighborhoods of the identity in $\operatorname{Homeo}_{K}\left(\kappa^{n}\right)=\operatorname{Homeo}(K)$ for some special compact open subsets $K \subseteq \kappa^{n}$. To that end, we describe finite clopen partitions of spaces of the form $\lambda_{1} \times \cdots \times \lambda_{n}$, where $\lambda_{i}$ are ordinals with $\operatorname{cf}\left(\lambda_{i}\right) \neq \omega$. (Observe that the space $\lambda_{1} \times \cdots \times \lambda_{n}$ is long if and only if $\operatorname{cf}\left(\lambda_{i}\right) \neq \omega$ for every $i$.) To that end, we introduce the notion of a grid partition.

Definition 5.7. Let $\lambda_{1}, \ldots, \lambda_{n}$ be ordinals, and put $X=\lambda_{1} \times \cdots \times \lambda_{n}$.

(a) For a finite subset $F_{i}=\left\{x_{1}<\cdots<x_{N}\right\} \subseteq \lambda_{i}$, the grid partition of $\lambda_{i}$ with respect to $F_{i}$ is

$$
\left.\mathcal{G}_{F_{i}}^{\lambda_{i}}:=\left\{\left[0, x_{1}\right]\right\} \cup\left\{\left[x_{j}+1, x_{j+1}\right] \mid j=1, \ldots, N-1\right\} \cup\left\{\left[x_{N}+1, \cdot\right)\right\}\right],
$$

where $[\alpha, \beta]:=\left\{x \in \lambda_{i} \mid \alpha \leq x \leq \beta\right\}$, and $[\alpha, \cdot):=\left\{x \in \lambda_{i} \mid \alpha \leq x\right\}$.

(b) For finite subsets $F_{i} \subseteq \lambda_{i}(i=1, \ldots, n)$, the grid partition of $X$ with respect to $\left\{F_{i}\right\}_{i=1}^{n}$ is

$$
\mathcal{G}_{\left\{F_{i}\right\}_{i=1}^{n}}^{X}:=\left\{\prod_{i=1}^{n} U_{i} \mid U_{i} \in \mathcal{G}_{F_{i}}^{\lambda_{i}}\right\} .
$$

Theorem 5.8. Let $\lambda_{1}, \ldots, \lambda_{n}$ be ordinals with $\operatorname{cf}\left(\lambda_{i}\right) \neq \omega$, and put $X=\lambda_{1} \times \cdots \times \lambda_{n}$. For every finite clopen partition $\mathcal{A}$ of $X$ there are finite subsets $F_{i} \subseteq \lambda_{i}$ such that $\mathcal{G}_{\left\{F_{i}\right\}_{i=1}^{n}}$ is a refinement of $\mathcal{A}$.

In order to prove Theorem 5.8, we first need a lemma.

Lemma 5.9. Let $\lambda_{1}, \ldots, \lambda_{n}$ be ordinals, put $X=\lambda_{1} \times \cdots \times \lambda_{n}$, and let $K \subseteq X$ be a non-empty compact subset. Then:

(a) $K$ has a maximal element with respect to the lexicographic order on $X$; and 
(b) if, in addition, $K$ is open, then $K$ is the disjoint union of finitely many compact open sets of the form $\prod_{i=1}^{n}\left[x_{i}, y_{i}\right]$, where for each $i$, either $x_{i}=0$ or $x_{i}$ is a successor ordinal.

Proof. Let $\preceq$ denote the lexicographic order on $X$.

(a) Let $\pi_{i}: X \rightarrow \lambda_{i}$ denote the canonical projection for $i=1, \ldots, n$. We construct $K_{1}, \ldots, K_{n+1}$ inductively. Put $K_{1}=K$. Suppose that the compact non-empty sets $K_{1}, \ldots, K_{i}$ have already been constructed. The set $\pi_{i}\left(K_{i}\right)$ is compact and non-empty, and so $a_{i}:=\max \pi_{i}\left(K_{i}\right)$ exists. Put

$$
K_{i+1}:=\pi_{i}^{-1}\left(\left\{a_{i}\right\}\right) \cap K_{i}
$$

Then $K_{n+1}=\left\{\left(a_{1}, \ldots, a_{n}\right)\right\}$ is a singleton, and $\left(a_{1}, \ldots, a_{n}\right)=\max (K, \preceq)$.

(b) Let $a=\left(a_{1}, \ldots, a_{n}\right) \in K$ be the maximal element of $K$ with respect to $\preceq$. Since $K$ is open, there is $x=\left(x_{1}, \ldots, x_{n}\right) \in X$ such that $a \in \prod_{i=1}^{n}\left[x_{i}, a_{i}\right] \subseteq K$, and either $x_{i}=0$ or $x_{i}$ is a successor ordinal. The set $U=\prod_{i=1}^{n}\left[x_{i}, a_{i}\right]$ is clopen, and so $K^{\prime}:=K \backslash U$ is again compact open. If $K^{\prime}=\emptyset$, then we are done. Otherwise, $\max \left(K^{\prime}, \preceq\right) \supsetneqq \max (K, \preceq)$, and one may repeat the same argument for $K^{\prime}$. Since each $\lambda_{i}$ is well-ordered, so is $(X, \preceq)$. Therefore, this process will terminate after finitely many steps.

We are now ready to prove Theorem 5.8 .

Proof of TheOrem 5.8. Let $\mathcal{A}=\left\{A_{1}, \ldots, A_{m}\right\}$ be a finite clopen partition of $X$. Then

$$
\mathcal{A}^{\prime}:=\left\{\operatorname{cl}_{\beta X} A_{1}, \ldots, \operatorname{cl}_{\beta X} A_{m}\right\}
$$

is a partition of $\beta X$ into compact open subsets.

In order to apply Lemma 5.9, we show that $\beta X$ is a product of ordinals. Each space $\lambda_{i}$ is sequentially compact, because $\operatorname{cf}\left(\lambda_{i}\right) \neq \omega$. Thus, the product $X=\prod_{i=1}^{n} \lambda_{i}$ is sequentially compact, and in particular, it is pseudocompact (cf. [5, 3.10.35, 3.10.30]). Therefore, by Glicksberg's Theorem ([7, Theorem 1]),

$$
\beta X=\prod_{i=1}^{n} \beta \lambda_{i}
$$

If $\operatorname{cf}\left(\lambda_{i}\right)=1$, then $\beta \lambda_{i}=\lambda_{i}$, and if $\operatorname{cf}\left(\lambda_{i}\right)>\omega$, then $\beta \lambda_{i}=\lambda_{i}+1$ ([6, 5N1]). Consequently, $\beta X$ is also a product of ordinals.

By Lemma 5.9, each $\operatorname{cl}_{\beta X} A_{j}$ is the disjoint union of compact open sets of the form $\prod_{i=1}^{n}\left[x_{i}, y_{i}\right]$, where for each $i$, either $x_{i}=0$ or $x_{i}$ is a successor ordinal. Thus, $\mathcal{A}^{\prime}$ has a compact open refinement

$$
\mathcal{A}^{\prime \prime}:=\left\{\prod_{i=1}^{n}\left[x_{i}^{(1)}, y_{i}^{(1)}\right], \ldots, \prod_{i=1}^{n}\left[x_{i}^{(l)}, y_{i}^{(l)}\right]\right\}
$$


where $x_{i}^{(j)}=0$ or $x_{i}^{(j)}$ is a successor ordinal for every $i$ and $j$. Put

$$
F_{i}:=\left\{y_{i}^{(j)} \mid j=1, \ldots, l \text { and } y_{i}^{(j)}<\lambda_{i}\right\}
$$

We claim that the grid partition $\mathcal{G}_{\left\{F_{i}\right\}_{i=1}^{n}}^{\beta X}$ is a refinement of $\mathcal{A}^{\prime \prime}$. Let $M \in \mathcal{G}_{\left\{F_{i}\right\}_{i=1}^{n}}^{\beta X}$. Then there are $\alpha=\left(\alpha_{1}, \ldots, \alpha_{n}\right)$ and $\gamma=\left(\gamma_{1}, \ldots, \gamma_{n}\right)$ in $\beta X$ such that $M=\prod_{i=1}^{n}\left[\alpha_{i}, \gamma_{i}\right]$. Since $\mathcal{A}^{\prime \prime}$ covers $\beta X$, there is $k$ such that $\alpha \in \prod_{i=1}^{n}\left[x_{i}^{(k)}, y_{i}^{(k)}\right]$. We show that $M \subseteq \prod_{i=1}^{n}\left[x_{i}^{(k)}, y_{i}^{(k)}\right]$. To that end, it suffices to prove that $\gamma \in \prod_{i=1}^{n}\left[x_{i}^{(k)}, y_{i}^{(k)}\right]$, or equivalently, that $\gamma_{i} \leq y_{i}^{(k)}$ for every $i$. Let $1 \leq i \leq n$. If $\alpha_{i}=0$, then $\gamma_{i}=\min F_{i}$ (or $\gamma_{i}=\lambda_{i}$ if $F_{i}=\emptyset$ ), and thus $\gamma_{i} \leq y_{i}^{(k)}$. Otherwise, $\alpha_{i}=y_{i}^{(r)}+1$ for some $r$, and $\gamma_{i}$ is the next element of $F_{i}$ (or $\gamma_{i}=\lambda_{i}$ ), and thus again $\gamma_{i} \leq y_{i}^{(k)}$.

This shows that $\mathcal{G}_{\left\{F_{i}\right\}_{i=1}^{n}}^{\beta X}$ is a refinement of $\mathcal{A}^{\prime \prime}$. Since $F_{i} \subseteq \lambda_{i}$ for every $i$, it follows that $\mathcal{G}_{\left\{F_{i}\right\}_{i=1}^{n}}^{X}$ is a refinement of $\mathcal{A}$.

The last step before proving part (c) of Theorem $\mathrm{D}$ is a technical proposition that allows one to use grid partitions with a fixed number of points in dealing with open subsets of the colimit space topology on $\underset{K \in \mathscr{K}(X)}{\operatorname{colim}} \operatorname{Homeo}_{K}(X)$.

Proposition 5.10. Let $\lambda_{1}, \ldots, \lambda_{n}$ be ordinals with $\operatorname{cf}\left(\lambda_{i}\right) \neq \omega$, and put $X=\prod_{i=1}^{n} \lambda_{i}$, and let $W$ be an open set containing the identity in the colimit space topology on $\underset{K \in \mathscr{K}(X)}{\operatorname{colim}} \operatorname{Homeo}_{K}(X)$. Then there are $m_{1}, \ldots, m_{n} \in \mathbb{N}$ and $\alpha^{(1)} \in X$ such that for every $\alpha=\left(\alpha_{1}, \ldots, \alpha_{n}\right) \geq \alpha^{(1)}$, there are $F_{i}^{\alpha} \subseteq \alpha_{i}$ such that $\left|F_{i}^{\alpha}\right|=m_{i}$ and

$$
U\left(\mathcal{G}_{\left\{F_{i}^{\alpha}\right\}_{i=1}^{n}}^{\downarrow \alpha}\right) \subseteq W \cap \operatorname{Homeo}_{\downarrow \alpha}(X)
$$

Proof. Since $W$ is open in the colimit space topology, the intersection $W \cap \mathrm{Homeo}_{\downarrow \alpha}(X)$ is open in $\operatorname{Homeo}_{\downarrow \alpha}(X)$ for every $\alpha \in X$. One has

$$
\operatorname{Homeo}_{\downarrow \alpha}(X) \cong \operatorname{Homeo}(\downarrow \alpha)=\operatorname{Homeo}_{c p t}(\downarrow \alpha),
$$

because $\downarrow \alpha$ is a compact open subset of the zero-dimensional space $X$. Thus, by Lemma 5.5, for every $\alpha \in X$ there is a finite clopen partition $\mathcal{A}_{\alpha}$ of $\downarrow \alpha$ such that $U\left(\mathcal{A}_{\alpha}\right) \subseteq W \cap \operatorname{Homeo}_{\downarrow \alpha}(X)$. By Theorem 5.8 applied to $X=\downarrow \alpha$, each $\mathcal{A}_{\alpha}$ has a grid partition refinement $\mathcal{G}_{\left\{E_{i}^{\alpha}\right\}_{i=1}^{n}}^{\downarrow \alpha}$ with $E_{i} \subseteq \alpha_{i}$, and consequently $U\left(\mathcal{G}_{\left\{E_{i}^{\alpha}\right\}_{i=1}^{n}}^{\downarrow \alpha}\right) \subseteq W \cap \operatorname{Homeo}_{\downarrow \alpha}(X)$.

We show now that it is possible to choose the grid points $E_{i}^{\alpha}$ in a way that the sizes of $E_{i}^{\alpha}$ stabilize. To that end, let $\preceq$ denote the lexicographic order on $\mathbb{N}^{n}$. For every $\alpha \in X$, put

$$
\left(m_{1}^{\alpha}, \ldots, m_{n}^{\alpha}\right):=\min _{\preceq}\left\{\left(\left|E_{1}^{\alpha}\right|, \ldots,\left|E_{n}^{\alpha}\right|\right) \mid E_{i}^{\alpha} \subseteq \alpha_{i}, U\left(\mathcal{G}_{\left\{E_{i}^{\alpha}\right\}_{i=1}^{n}}^{\downarrow \alpha}\right) \subseteq W \cap \operatorname{Homeo}_{\downarrow \alpha}(X)\right\} .
$$


We show that the map $f: X \rightarrow\left(\mathbb{N}^{n}, \preceq\right)$ defined by $\alpha \mapsto\left(m_{1}^{\alpha}, \ldots, m_{n}^{\alpha}\right)$ is monotone. Let $\alpha \leq \beta \in X$, and pick $F_{i}^{\beta} \subseteq \beta_{i}$ such that $\left|F_{i}^{\beta}\right|=m_{i}^{\beta}$ and $U\left(\mathcal{G}_{\left\{F_{i}^{\beta}\right\}_{i=1}^{n}}^{\downarrow \beta}\right) \subseteq W \cap \operatorname{Homeo}_{\downarrow \beta}(X)$. For $E_{i}^{\alpha}=F_{i}^{\beta} \cap \alpha_{i}$, one has $U\left(\mathcal{G}_{\left\{E_{i}^{\alpha}\right\}_{i=1}^{n}}^{\nu^{\prime}}\right) \subseteq W \cap \operatorname{Homeo}_{\downarrow \alpha}(X)$ and $\left|E_{i}^{\alpha}\right| \leq\left|F_{i}^{\beta}\right|=m_{i}^{\beta}$ for every $i$. In particular,

$$
\left(m_{1}^{\alpha}, \ldots, m_{n}^{\alpha}\right) \preceq\left(\left|E_{1}^{\alpha}\right|, \ldots,\left|E_{n}^{\alpha}\right|\right) \preceq\left(\left|F_{1}^{\alpha}\right|, \ldots,\left|F_{n}^{\alpha}\right|\right)=\left(m_{1}^{\beta}, \ldots, m_{n}^{\beta}\right) .
$$

Since $\operatorname{cf}\left(\lambda_{i}\right) \neq \omega$, the directed set $(X, \leq)$ is long, and thus so is its image $f(X)$ under the orderpreserving map $f$. Therefore, the countable set $f(X)$ has an upper bound $\left(m_{1}, \ldots, m_{n}\right) \in f(X)$, and it is the maximal element of $f(X)$. Pick $\alpha^{(1)} \in X$ such that $f\left(\alpha^{(1)}\right)=\left(m_{1}, \ldots, m_{n}\right)$. Then for every $\alpha \geq \alpha^{(1)}$, one has $m_{i}^{\alpha}=m_{i}$ for every $i$. Hence, one may pick $F_{i}^{\alpha} \subseteq \alpha_{i}$ such that $\left|F_{i}^{\alpha}\right|=m_{i}$ and $U\left(\mathcal{G}_{\left\{F_{i}^{\alpha}\right\}_{i=1}^{n}}^{\downarrow \beta}\right) \subseteq W \cap \operatorname{HomeO}_{\downarrow \alpha}(X)$.

We turn now to the proof of part (c) of Theorem $\mathrm{D}$. We will show that when the $\lambda_{i}$ are equal to a regular uncountable cardinal $\kappa$, then not only do the cardinalities of the $F_{i}^{\alpha}$ stabilize, but also the sets themselves do. To do so, recall that a subset $S$ of a cardinal $\kappa$ is called stationary if it intersects every closed and unbounded subset of $\kappa$. Fodor's Pressing Down Lemma states that:

Theorem 5.11. ([11, Theorem 8.7]) Let $\kappa$ be a regular uncountable cardinal, and let $S \subseteq \kappa$ be a stationary subset. If $f: S \rightarrow \kappa$ satisfies $f(x)<x$ for every $x \in S$, then there is a stationary set $T \subseteq S$ such that $f_{\mid T}$ is constant.

Proof of Theorem $\mathbf{D}(\mathbf{C})$. By Corollary 5.4, it suffices to prove the statement for $X=\kappa^{n}$, where $\kappa$ is a regular uncountable cardinal. The colimit space topology on $\underset{K \in \mathscr{K}(X)}{\operatorname{colim}} \operatorname{Homeo}_{K}(X)$ is finer than the topology of $\mathrm{Homeo}_{c p t}(X)$. We show the converse.

Let $W$ be an open subset of $\underset{K \in \mathscr{K}(X)}{\operatorname{colim}} \operatorname{Homeo}_{K}(X)$ and let $h \in W$. Since translation is continuous in the colimit space topology, $W_{0}:=W h^{-1}$ is an open set containing the identity. Thus, by Proposition 5.10, there are $m_{1}, \ldots, m_{n} \in \mathbb{N}$ and $\alpha^{(1)} \in X$ such that for every $\alpha \geq \alpha^{(1)}$ there are $F_{i}^{\alpha} \subseteq \alpha$ with $\left|F_{i}^{\alpha}\right|=m_{i}$ and

$$
U\left(\mathcal{G}_{\left\{F_{i}^{\alpha}\right\}_{i=1}^{n}}^{\downarrow \alpha}\right) \subseteq W_{0} \cap \operatorname{Homeo}_{\downarrow \alpha}(X)
$$

Let $\Delta: \kappa \rightarrow \kappa^{n}$ denote the diagonal map, and put $\gamma^{(1)}=\max _{1 \leq i \leq n} \alpha_{i}^{(1)}$, where $\alpha^{(1)}=\left(\alpha_{1}^{(1)}, \ldots, \alpha_{n}^{(1)}\right)$. For $\gamma \geq \gamma^{(1)}$, for every $(i, j)$ such that $1 \leq i \leq n$ and $1 \leq j \leq m_{i}$, let $f_{i, j}(\gamma)$ denote the $j$-th point in $F_{i}^{\Delta \gamma}$. Since $F_{i}^{\Delta \gamma} \subseteq(\Delta \gamma)_{i}=\gamma$, one has $f_{i, j}(\gamma)<\gamma$. By repeated application of Theorem 5.11, one obtains a stationary set $T \subseteq\left[\gamma^{(1)}, \kappa\right)$ such that $f_{i, j \mid T}$ is constant for every $1 \leq i \leq n$ and $1 \leq j \leq m_{i}$. In other words, there are $F_{i} \subseteq \kappa$ such that $F_{i}^{\Delta \gamma}=F_{i}$ for every $\gamma \in T$. We show that

$$
U\left(\mathcal{G}_{\left\{F_{i}\right\}_{i=1}^{n}}^{X}\right) \subseteq W_{0}
$$

Let $g \in U\left(\mathcal{G}_{\left\{F_{i}\right\}_{i=1}^{n}}^{\text {nat }}\right)$. Since $g$ has compact support, there is $\beta$ such that $g \in \operatorname{Homeo}_{\downarrow} \Delta \beta(X)$. Since $T$ is stationary, in particular, it intersects $[\beta, \kappa)$, and so there is $\gamma \in T$ such that $\beta \leq \gamma$. Therefore,

$$
g \in U\left(\mathcal{G}_{\left\{F_{i}\right\}_{i=1}^{n}}^{X}\right) \cap \text { Homeo }_{\downarrow \Delta \gamma}(X)=U\left(\mathcal{G}_{\left\{F_{i}^{\Delta \gamma}\right\}_{i=1}^{\natural}}^{\downarrow \gamma} \stackrel{\sqrt[60]{\subseteq}}{\subseteq} W_{0} .\right.
$$

Hence, $U\left(\mathcal{G}_{\left\{F_{i}\right\}_{i=1}^{n}}^{X}\right) h \subseteq W$, and $W$ is open in the topology of $\operatorname{Homeo}_{c p t}(X)$. 


\section{Acknowledgments}

We would like to express our heartfelt gratitude to Karl H. Hofmann for introducing us to each other, and to the organizers of the 2015 Summer Conference on Topology and its Applications for providing an environment conducive for this collaboration to form. We wish to thank Frédéric Mynard for the valuable correspondence. We are grateful to Karen Kipper for her kind help in proofreading this paper for grammar and punctuation. We are grateful to the anonymous referee for their detailed and helpful suggestions that have contributed to the articulation and clarity of the manuscript.

\section{References}

[1] R. Arens. Topologies for homeomorphism groups. American Journal of Mathematics, 68(4):593-610, 1946.

[2] A. V. Arhangel'skii. The frequency spectrum of a topological space and the product operation. Trans. Moscow Math. Soc., Issue 2:163-200, 1981.

[3] T. M. Bisgaard. The topology of finitely open sets is not a vector space topology. Arch. Math. (Basel), 60(6):546$552,1993$.

[4] J. Dieudonné. Sur la complétion des groupes topologiques. CR Acad. Sci. Paris, 218:774-776, 1944.

[5] R. Engelking. General topology. PWN—Polish Scientific Publishers, Warsaw, 1977. Translated from the Polish by the author, Monografie Matematyczne, Tom 60. [Mathematical Monographs, Vol. 60].

[6] L. Gillman and M. Jerison. Rings of continuous functions. The University Series in Higher Mathematics. D. Van Nostrand Co., Inc., Princeton, N.J.-Toronto-London-New York, 1960.

[7] I. Glicksberg. Stone-Čech compactifications of products. Trans. Amer. Math. Soc., 90:369-382, 1959.

[8] H. Glöckner, R. Gramlich, and T. Hartnick. Final group topologies, Kac-Moody groups and Pontryagin duality. Israel J. Math., 177:49-101, 2010.

[9] H. Herrlich. Separation axioms and direct limits. Canadian Mathematical Bulletin, 12(3):337-338, 1969.

[10] T. Hirai, H. Shimomura, N. Tatsuuma, and E. Hirai. Inductive limits of topologies, their direct products, and problems related to algebraic structures. J. Math. Kyoto Univ., 41(3):475-505, 2001.

[11] T. Jech. Set Theory. Perspectives in Mathematical Logic. Springer Berlin Heidelberg, 2013.

[12] I. Juhász, J. van Mill, and W. Weiss. Variations on w-boundedness. Isr. J. Math., 194:745-766, 2013.

[13] P. J. Nyikos. Chapter 25 - first countable, countably compact, noncompact spaces. In E. Pearl, editor, Open Problems in Topology II, pages 217-224. Elsevier, Amsterdam, 2007.

[14] H. H. Schaefer and M. P. Wolff. Topological vector spaces, volume 3 of Graduate Texts in Mathematics. Springer-Verlag, New York, second edition, 1999.

[15] A. Yamasaki. Inductive limit of general linear groups. J. Math. Kyoto Univ., 38(4):769-779, 1998.

Rafael Dahmen

Department of Mathematics

Karlsruhe Institute of Technology

D-76128 Karlsruhe

Germany

rafael.dahmen@kit.edu
Gábor Lukács

Department of Mathematics and Statistics

Dalhousie University

Halifax, B3H 3J5, Nova Scotia

Canada

lukacs@topgroups.ca 